\title{
The Interspecific Fungal Hybrid Verticillium longisporum Displays Subgenome-Specific Gene Expression
}

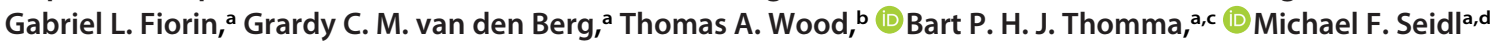 \\ aLaboratory of Phytopathology, Wageningen University and Research, Wageningen, The Netherlands \\ bDepartment of Crops and Agronomy, National Institute of Agricultural Botany, Cambridge, United Kingdom \\ cUniversity of Cologne, Institute for Plant Sciences, Cluster of Excellence on Plant Sciences (CEPLAS), Cologne, Germany \\ dTheoretical Biology and Bioinformatics, Utrecht University, Utrecht, The Netherlands \\ eDepartamento de Biología Celular, Genética y Fisiología, Universidad de Málaga, Málaga, Spain \\ Fabian van Beveren, Luis Rodriguez-Moreno, H. Martin Kramer, and Edgar A. Chavarro Carrero contributed equally to this work. \\ Thomas A. Wood, Bart P. H. J. Thomma, and Michael F. Seidl contributed equally to this work.
}

Jasper R. L. Depotter, ${ }^{a, b, c}$ Fabian van Beveren, ${ }^{a}$ Luis Rodriguez-Moreno, ${ }^{a, e}$ H. Martin Kramer, ${ }^{a}$ Edgar A. Chavarro Carrero, ${ }^{a}$

ABSTRACT Hybridization is an important evolutionary mechanism that can enable organisms to adapt to environmental challenges. It has previously been shown that the fungal allodiploid species Verticillium longisporum, the causal agent of verticillium stem striping in rapeseed, originated from at least three independent hybridization events between two haploid Verticillium species. To reveal the impact of genome duplication as a consequence of hybridization, we studied the genome and transcriptome dynamics upon two independent $V$. longisporum hybridization events, represented by the hybrid lineages "A1/D1" and "A1/D3." We show that V. longisporum genomes are characterized by extensive chromosomal rearrangements, including between parental chromosomal sets. $V$. longisporum hybrids display signs of evolutionary dynamics that are typically associated with the aftermath of allodiploidization, such as haploidization and more relaxed gene evolution. The expression patterns of the two subgenomes within the two hybrid lineages are more similar than those of the shared A1 parent between the two lineages, showing that the expression patterns of the parental genomes homogenized within a lineage. However, as genes that display differential parental expression in planta do not typically display the same pattern in vitro, we conclude that subgenome-specific responses occur in both lineages. Overall, our study uncovers genomic and transcriptomic plasticity during the evolution of the filamentous fungal hybrid $V$. longisporum and illustrates its adaptive potential.

IMPORTANCE Verticillium is a genus of plant-associated fungi that includes a few plant pathogens that collectively affect a wide range of hosts. On several occasions, haploid Verticillium species hybridized into the stable allodiploid species Verticillium longisporum, which is, in contrast to haploid Verticillium species, a Brassicaceae specialist. Here, we studied the evolutionary genome and transcriptome dynamics of $V$. longisporum and the impact of the hybridization. V. longisporum genomes display a mosaic structure due to genomic rearrangements between the parental chromosome sets. Similar to other allopolyploid hybrids, V. longisporum displays an ongoing loss of heterozygosity and more relaxed gene evolution. Also, differential parental gene expression is observed, with enrichment for genes that encode secreted proteins. Intriguingly, the majority of these genes display subgenome-specific responses under differential growth conditions. In conclusion, hybridization has incited the genomic and transcriptomic plasticity that enables adaptation to environmental changes in a parental allele-specific fashion.
Citation Depotter JRL, van Beveren F, Rodriguez-Moreno L, Kramer HM, Chavarro Carrero EA, Fiorin GL, van den Berg GCM, Wood TA, Thomma BPHJ, Seidl MF. 2021. The interspecific fungal hybrid Verticillium longisporum displays subgenome-specific gene expression. mBio 12:e01496-21. https:// doi.org/10.1128/mBio.01496-21.

Editor Joseph Heitman, Duke University Copyright $\odot 2021$ Depotter et al. This is an open-access article distributed under the terms of the Creative Commons Attribution 4.0 International license.

Address correspondence to Bart P. H. J. Thomma, bthomma@uni-koeln.de, or Michael F. Seidl, m.f.seidl@uu.nl.

Received 25 May 2021

Accepted 9 June 2021

Published 20 July 2021 
KEYWORDS allopolyploidization, Verticillium stem striping, genome rearrangements, gene conversion, haploidization, mosaic genome, chromatin conformation capture $(\mathrm{Hi}-\mathrm{C})$

pon hybridization, two distinct genotypes are merged into a single organism. This surge in genomic variation can increase the adaptive potential of hybrid organisms, which may explain why stable hybrids are generally more fit than their parents in particular environments (1). However, hybrids may also encounter incompatibilities between parental genomes as they lack the recently shared evolutionary history (2). Hybridization can lead to the emergence of new species that are reproductively isolated from their parents, known as hybrid speciation $(3,4)$. Although the incidence of hybridization may be rare due to such incompatibilities, many organisms encountered hybridization at a particular point in their evolution (5). Hybridization has also impacted the evolution of humans, as our genomes still contain traces of Neanderthal introgression (6). Hybridization can occur between gametes after conventional meiosis, leading to so-called homoploid hybrids. Alternatively, when complete sets of parental chromosomes combine, hybridization is accompanied by genome duplication during so-called allopolyploidization.

Hybridization has impacted the evolution of a wide diversity of fungi (7-9). For instance, the yeast Saccharomyces paradoxus, a close relative of the baker's yeast Saccharomyces cerevisiae, has naturally hybridized in North American forests (10), whereas S. cerevisiae itself was also shown to have undergone an ancient interspecies hybridization event (11). Similarly, various Candida species that are opportunistic human pathogens display genomic traces of hybridization events (12-15). Hybridization also contributed to the evolution of various plant-pathogenic fungi (7). Plant pathogens generally coevolve with their hosts to evade host immunity, while hosts attempt to intercept pathogen ingress (16). In this process, plant pathogens secrete effector proteins that contribute to host immunity evasion and interfere with host metabolic processes (17) or affect other processes to contribute to host colonization (18), such as the manipulation of host microbiomes $(19,20)$. Due to the increased adaptation potential, hybridization has been proposed as a potent driver in pathogen evolution as it can impact host interactions through increased virulence and host range alterations (8). For instance, the Ug99 strain of the wheat stem rust pathogen Puccinia graminis f. sp. tritici arose from a hybridization event and caused devastating epidemics in Africa and the Middle East $(21,22)$. Recent hybridization between wheat powdery mildew, Blumeria graminis f. sp. tritici, and rye powdery mildew, $B$. graminis $\mathrm{f}$. sp. secalis, gave rise to the novel mildew species $B$. graminis f. sp. triticale, which, in contrast to its parents, is able to cause disease on triticale (23).

Upon hybridization, genomes typically experience a so-called "genome shock," inciting major genomic reorganizations that can manifest as genome rearrangements, extensive gene loss, transposon activation, and alterations in gene expression $(24,25)$. Conceivably, these early-stage alterations are primordial for hybrid survival, as divergent evolution is principally associated with incompatibilities between the parental genomes (26). Additionally, these initial reorganizations and further alterations in the aftermath of hybridization provide a source for environmental adaptation. Frequently, hybrid genomes lose their heterozygosity over time (27). Hybrids that are still sexually compatible with one of their parents can lose heterozygosity through backcrossing. Alternatively, heterozygosity can be a result of the direct loss of a homolog of one of the two parents (i.e., a homeolog) through deletion or gene conversion whereby one of the copies replaces its homeologous counterpart. Gene conversion and the homogenization of complete chromosomes played a pivotal role in the evolution of the osmotolerant yeast species Pichia sorbitophila (28). Two of its seven chromosome pairs consist of partly heterozygous and partly homozygous sections, whereas two chromosome pairs are completely homozygous. Gene conversion may eventually result in chromosomes consisting of sections of both parental origins, so-called "mosaic 
genomes" (29). However, mosaic genomes can also arise through recombination between chromosomes of the different parents, such as in the hybrid yeast Zygosaccharomyces parabailii (30). Hybridization associated with polyploidy, allopolyploids, can have additional adaptive potential through the presence of an additional copy for most genes, which gives leeway to functional diversification $(31,32)$. Hybridization typically also entails alterations of gene expression patterns that are nonadditive from the parental expression patterns $(33,34)$. Nevertheless, expression patterns are generally conserved upon hybridization, as the majority of allopolyploid genes are expressed in a fashion similar to that of their parental orthologs (35). For instance, more than half of the genes in an allopolyploid strain of the fungal grass endophyte Epichloë retained their parental gene expression pattern (36). Similar conservation has also been observed for Blumeria graminis f. sp. triticale as over half of the $5 \%$ most highly expressed genes are shared with both of its hybridization parents (37). In conclusion, the genomic and transcriptomic alterations accompanied by hybridization make hybrids have a high potential for environmental adaptation (8).

Within the Verticillium genus that comprises nine haploid species, hybridization resulted in the emergence of the species Verticillium longisporum (38-41). V. longisporum is subdivided into three lineages, each representing a separate hybridization event $(39,41)$. Verticillium species $A 1$ is a parent of each of the three hybrids and hybridized with Verticillium species D1, D2, and D3, resulting in the $V$. longisporum lineages A1/D1, $A 1 / D 2$, and A1/D3, respectively. Whereas species D2 and D3 have been classified as "likely Verticillium dahliae," species D1 has been classified as an enigmatic species that is closely related to $V$. dahliae (39). Species A1 is also an enigmatic species that diverged from $V$. dahliae earlier than the D1 species (39). Similar to the haploid Verticillium species, $V$. longisporum is thought to mainly undergo asexual reproduction, as a sexual cycle has never been described, and populations are not outcrossing (40, 41). Interestingly, V. longisporum mainly infects plant hosts of the Brassicaceae family, whereas other Verticillium species do not cause disease on brassicaceous hosts (42). Moreover, while $V$. dahliae is characterized by an extremely broad host range that comprises hundreds of (non-Brassicaceae) plant species, $V$. longisporum has only a limited host range and hardly infects non-Brassicaceae species (42). After hybridization, $V$. longisporum conceivably encountered extensive genetic and transcriptomic alterations that facilitated its viability as a hybrid and the shift toward brassicaceous hosts. In this study, we investigated the impact of allodiploidization on the evolution of $V$. longisporum by investigating genome, gene, and transcriptomic plasticity within and between two of the hybridization events.

\section{RESULTS}

Verticillium longisporum displays a mosaic genome structure. The genomes of three $V$. longisporum strains from two different hybridization events were analyzed to investigate the impact of hybridization on genome structure. Previously, V. longisporum strains VLB2 and VL20, both belonging to the A1/D1 hybridization event, were sequenced with the PacBio RSII platform and assembled de novo (40). We now additionally sequenced $V$. longisporum strain PD589, which originates from the A1/D3 hybridization event (39), using Oxford Nanopore Technologies (ONT) sequencing technology and the BGISeq platform to obtain long reads and paired-end short reads, respectively. All $V$. longisporum genome assemblies were improved using chromatin conformation capture (Hi-C) sequencing that detects DNA interactions (43) (see Fig. S1 in the supplemental material). Moreover, centromeres can be located with $\mathrm{Hi}-\mathrm{C}$ sequencing as they display strong interactions with centromeres in other chromosomes (44) (Fig. S1). We obtained genome assemblies of 72.7, 72.2, and 72.0 Mb consisting of 15, 15, and 16 pseudochromosomes for VLB2, VL20, and PD589, respectively (Fig. 1A and Table 1). Every pseudochromosome contained a centromere, suggesting that the A1/D1 isolates have 15 chromosomes and that the A1/D3 isolate PD589 contains 16 chromosomes (Fig. 1A). However, chromosome 13 of strain PD589 displayed 
A

= A1 sub-genome $\square=$ D1 sub-genome $\quad \square=$ D3 sub-genome

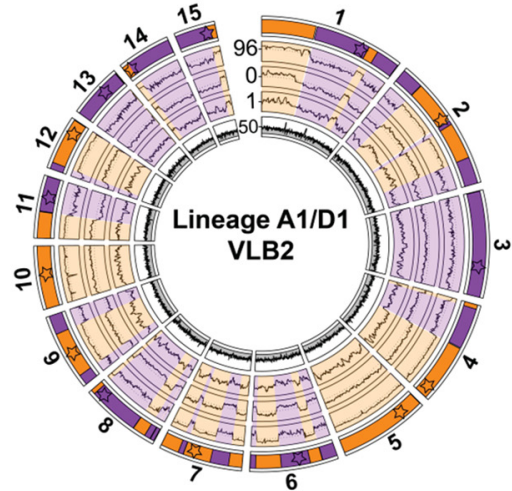

Centromere flanking regions $\begin{array}{llll}7 & 7 & 1\end{array}$

B

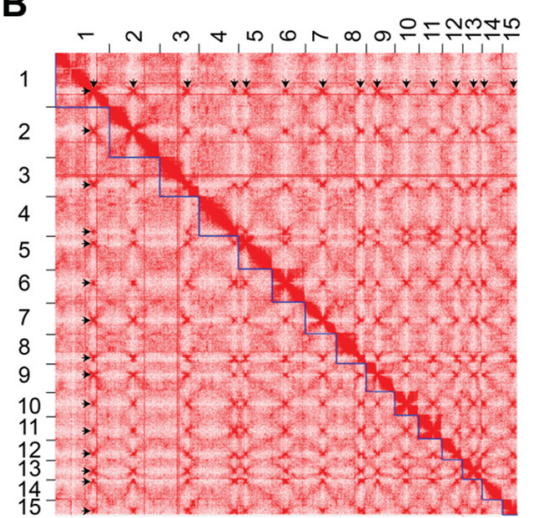

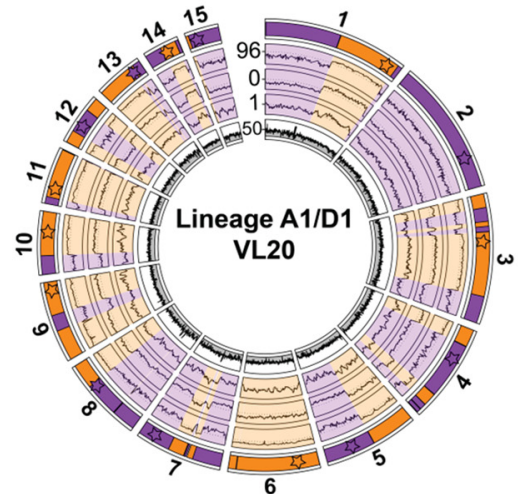

Centromere flanking regions 8070

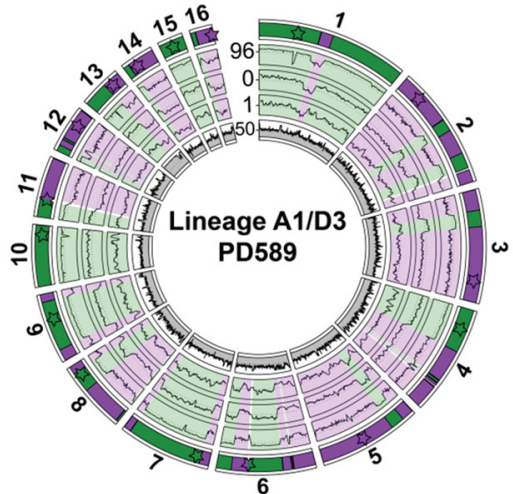

Centromere flanking regions $\begin{array}{lll}7 & 7 & 2\end{array}$

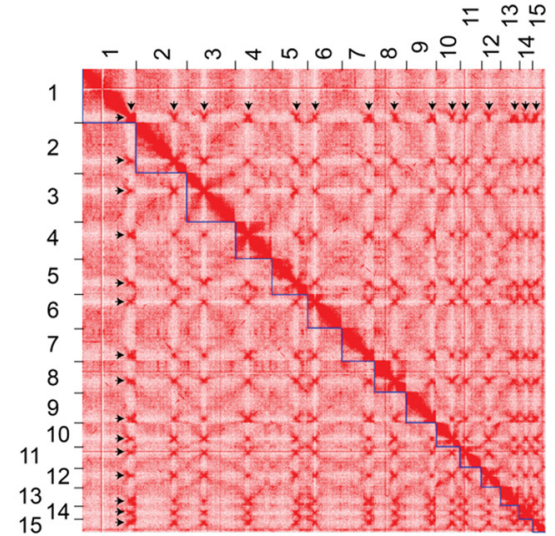

$=m$

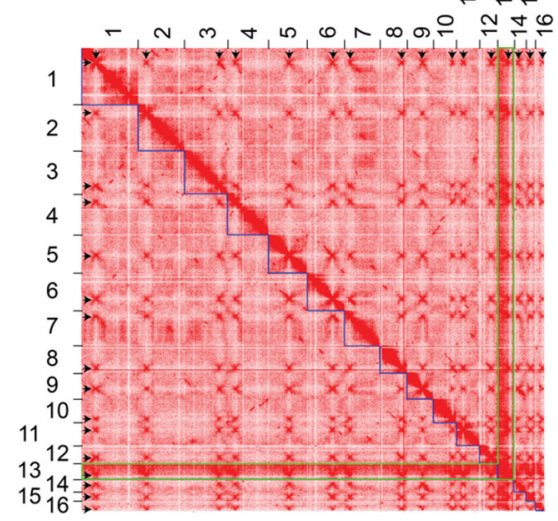

FIG 1 Verticillium longisporum displays a mosaic genome structure. (A) V. longisporum chromosomes of strains VLB2, VL20, and PD589. The different lanes in the circular plots represent (i) regions assigned to species A1, species D1, and species D3, with the stars representing the locations of the centromeres and their color representing the parental origin based on their flanking regions; (ii) sequence similarity of $V$. longisporum alignments to $V$. dahliae (percent identity); (iii) differences in sequence identity in percentage points ( $\mathrm{pp}$ ) between exonic regions of $V$. longisporum double-copy genes, where only gene pairs with an ortholog in $V$. dahliae are depicted and alleles with a higher identity to $V$. dahliae are depicted as a positive pp difference, whereas the corresponding homolog is depicted as a negative pp difference; (iv) the relative difference in GC content (dGC) between genes in double copy; and (v) read depth with nonoverlapping windows of $10 \mathrm{~kb}$. Data points of lanes 3 to 5 represent the average values for a window of 11 genes, which proceed with a step of 1 gene. (B) Hi-C contact frequency matrices for the three $V$. longisporum strains. Red indicates the contact intensity between genome regions, and the blue squares represent the pseudochromosomes. Centromeres display strong interchromosomal contacts and are visible as red dots outside the pseudochromosomes and indicated with black arrows. Pseudochromosome 13 of strain PD589 generally displays stronger interactions than the other pseudochromosomes and is outlined in green.

remarkably stronger DNA interactions than the other chromosomes (Fig. 1B, green outline), as the median read coverage of chromosome 13 is $110 \times$, whereas the read coverage is $58 \times$ to $70 \times$ for all other chromosomes (Fig. S2). This finding suggests that chromosome 13 recently (partly) duplicated since the high sequence identity of the duplicated regions resulted in a collapsed assembly. Consequently, strain PD589 may therefore actually have 17 chromosomes in total.

Being able to determine the parental origin of individual genomic regions is elementary to investigating genome evolution in the aftermath of hybridization. As the $D$ parents of $V$. longisporum hybridizations (D1 and D3) are phylogenetically more closely related to $V$. dahliae than parent $A 1$ (39), $V$. longisporum genome alignments to $V$. dahliae display a bimodal distribution with minima at $\sim 96.0 \%$ identity (Fig. S3A). Similarly, the sequence identity between coding regions of $V$. longisporum and $V$. dahliae orthologs displayed a bimodal distribution, with minima that are less pronounced than those of the genome alignments (Fig. S3B). To separate the two subgenomes, we used genome alignments and coding region sequence identities. Genome regions were assigned to parent $\mathrm{A} 1$ if their average sequence identity to $V$. dahliae was lower than 
TABLE 1 Comparison of Verticillium longisporum and Verticillium dahliae genome assemblies

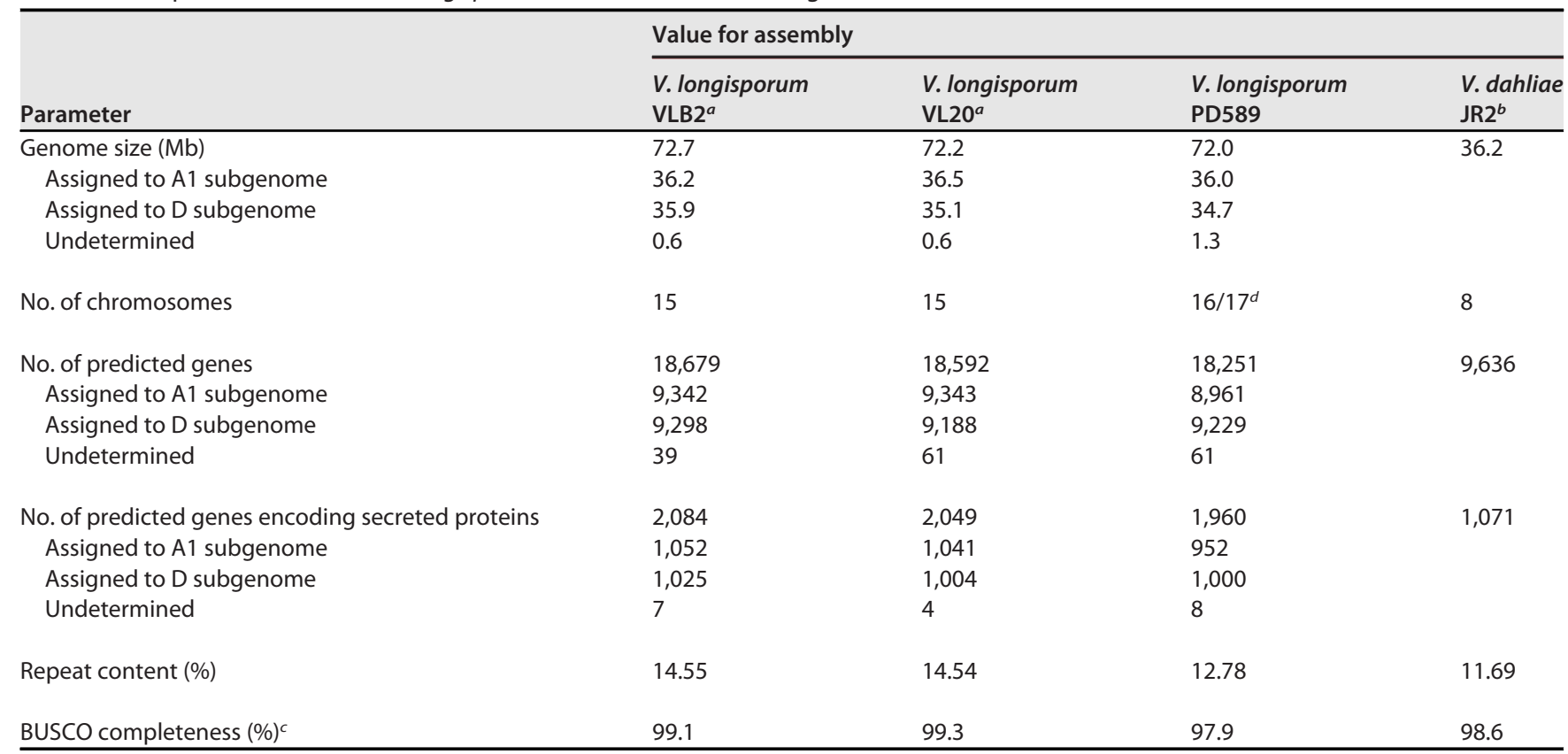

aPreviously published assemblies were reassembled using $\mathrm{Hi}-\mathrm{C}$ sequencing (40).

bSee reference 47.

cBased on Ascomycota benchmarking universal single-copy orthologs (BUSCOs).

वThe total chromosome number is uncertain as PD589 contains one (partially) duplicated chromosome.

this minimum to parent $\mathrm{A} 1$ and/or their coding regions displayed 93 to $98.5 \%$ sequence identity with their $V$. dahliae ortholog (Fig. S3). In contrast, regions with alignments and/or coding regions with higher sequence identities were assigned to the $\mathrm{D}$ parent (Fig. S3). In this manner, 36.0 to $36.5 \mathrm{Mb}$ were assigned to the $\mathrm{A} 1$ parents, and 34.7 to $35.9 \mathrm{Mb}$ were assigned to the $\mathrm{D}$ parents (Fig. $1 \mathrm{~A}$ and Table 1). Thus, the subgenome sizes are quite similar for each of the isolates and correspond to the expected genome sizes of haploid Verticillium species $(44,45)$. As the three V. longisporum strains have the $\mathrm{A} 1$ parent in common, we used nucleotide substitutions in the $\mathrm{A} 1$ coding regions to roughly estimate the minimum divergence time of the $V$. longisporum strains since their last common ancestor. Estimates of nucleotide mutation rates are as yet not available for Verticillium, so we used the estimated asexual mutation rate for the coding region of Neurospora crassa $\left(9.05 \times 10^{-9}\right.$ per site per day or $6.03 \times 10^{-10}$ per site per cell division) (46). Between strains VLB2 and VL20 from the same hybridization event, $0.073 \%$ of the nucleotides of their $A 1$ homologs displayed substitutions, which corresponds to $1,204,419$ cell divisions or 220 years of continuous growth on culture medium. In contrast, between hybridization events, PD589 homologs displayed 0.24 and $0.23 \%$ nucleotide substitutions with VLB2 and VL20 homologs, respectively, which correspond to $3,899,045$ and $3,845,760$ cell divisions or 712 and 702 years of continuous growth on culture medium, respectively. As Verticillium does not grow continuously under optimal conditions in nature and produces microsclerotia to overwinter in the absence of the host, which can remain dormant and viable for more than 10 years when hosts are not available in the next growing season (41), these divergence time estimates are certainly severe underestimations of the actual divergence times and should be considered minimum divergence times.

The majority of the $V$. longisporum chromosomes are composed of DNA regions that originate from different parents, and only two chromosomes have a single parental origin in each of the strains (Fig. 1; Table S1). Using different genome assembly approaches, the genomic regions of different parental origins were consistently assembled together, excluding the possibility that this phenomenon is caused by assembly artifacts (Fig. S4). Thus, V. longisporum chromosomes generally are mosaics of 
DNA regions of different parental origins. As genomic rearrangements may also occur over centromeres (44), we assessed if such rearrangements could be identified. One centromere of VLB2 and two of PD589 are flanked by regions of differential parental origins, demonstrating that rearrangements between the parental genomes occurred over these centromeres (Fig. 1A). In contrast, strain VL20 did not have centromeres flanked by regions of different parental origins (Fig. 1A).

The mitochondrial genome is inherited from the $\mathbf{A} 1$ parent in all lineages. To determine the phylogenetic position of the parental subgenomes of $V$. longisporum, we used the $V$. longisporum subgenome sequences and previously published genome sequences of the haploid Verticillium species $(45,47)$ to construct a phylogenetic tree based on 1,520 ascomycete benchmarking universal single-copy orthologs (BUSCOs) that were present in a single copy in all analyzed Verticillium lineages. In accordance with previous phylogenetic studies $(39,40)$, the A1 parents diverged earlier from $V$. dahliae than the D1 and D3 parents (Fig. S5). Furthermore, the D1 parent diverged earlier from $V$. dahliae than the D3 parent. We also constructed a phylogenetic tree based on mitochondrial DNA to determine the parental origin of the mitochondria. The $V$. longisporum mitochondrial genomes were assembled in a single contig with overlapping ends, indicating their circular nature. The mitochondrial genomes of the three $V$. longisporum strains were all $26.2 \mathrm{~kb}$ in size and were more than $99.9 \%$ identical in sequence. The phylogenetic position of the $V$. longisporum mitochondrial genomes clusters with the mitochondrial genomes of $V$. alfalfae and $V$. nonalfalfae (Fig. S5). As the mitochondrial genome sequences are almost identical for three strains that are derived from the two hybridization events, the common A1 parent is the likely donor of the mitochondria.

Genomic rearrangements are responsible for the mosaic nuclear genome. Typically, a mosaic structure of a hybrid nuclear genome can originate from gene conversion or chromosomal rearrangements between DNA strands of different parental origins (27). To analyze the extent of gene conversion, protein-coding genes were predicted for the $V$. longisporum strains using BRAKER with RNA sequencing (RNA-Seq) data from fungal cultures grown in vitro (48). The number of predicted genes ranged from 18,251 to 18,679 for the different $V$. longisporum strains, which is 89 to $94 \%$ higher than the gene number of $V$. dahliae strain JR2 predicted using the same methodology (9,636 genes) (Table 1). In total, 8,961 to 9,343 genes were assigned to the subgenome of parent $A 1$, whereas the number of genes in the D3 subgenomes ranged from 9,188 to 9,298 (Table 1). Thus, the gene numbers are similar for the different $V$. longisporum subgenomes and comparable to the gene number of $V$. dahliae. Over $79 \%$ of the $V$. longisporum genes have one homolog; i.e., they occur in two copies, which can originate from gene duplication (paralogy) or the hybridization event (homeology) (Fig. 2A and B). Within each of the $V$. longisporum subgenomes, most genes (96.9 to 99.6\%) have no additional homolog and occur in a single copy (Fig. 2B), indicating that most homologous gene pairs in each $V$. longisporum genome are homeologous in nature and that gene conversion played only a minor role after hybridization. Accordingly, over $80 \%$ of the D subgenomes have one homeologous genome region (Fig. 2C). To find traces of gene conversion during their evolution, the sequence identities of 6,213 genes that have two homologous copies in the two A1/D1 strains were compared, as these two strains belong to distinct populations (40). Only seven genes were found to be highly similar ( $<1 \%$ nucleotide sequence diversity) in VLB2, whereas the corresponding gene pair in VL20 was more diverse (>1\%) (Fig. 3A). Similarly, in V. longisporum strain VL20, four highly similar copies were found that are more divergent in VLB2, thereby confirming that gene conversion has hitherto played only a marginal role in the evolutionary aftermath of $V$. longisporum hybridization.

Considering that gene conversion played only a minor role during genome evolution (Fig. 3), the mosaic genome structure of $V$. longisporum likely originated from rearrangements between homeologous chromosomes. To identify chromosomal rearrangements after the hybridization event that led to the A1/D1 lineage, the genome of V. longisporum strain VLB2 was aligned to that of strain VL20, revealing 46 syntenic 
A

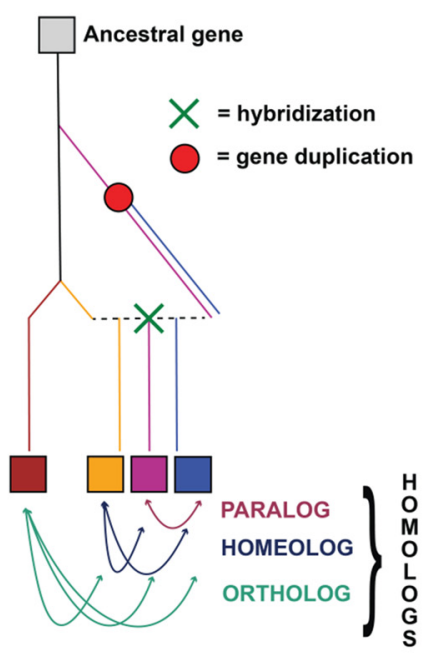

C

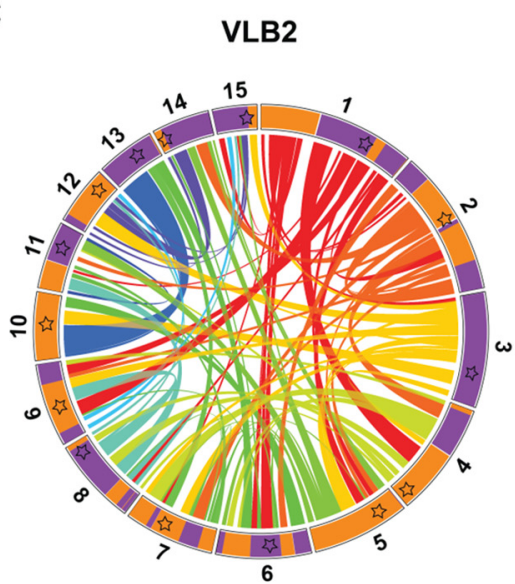

B

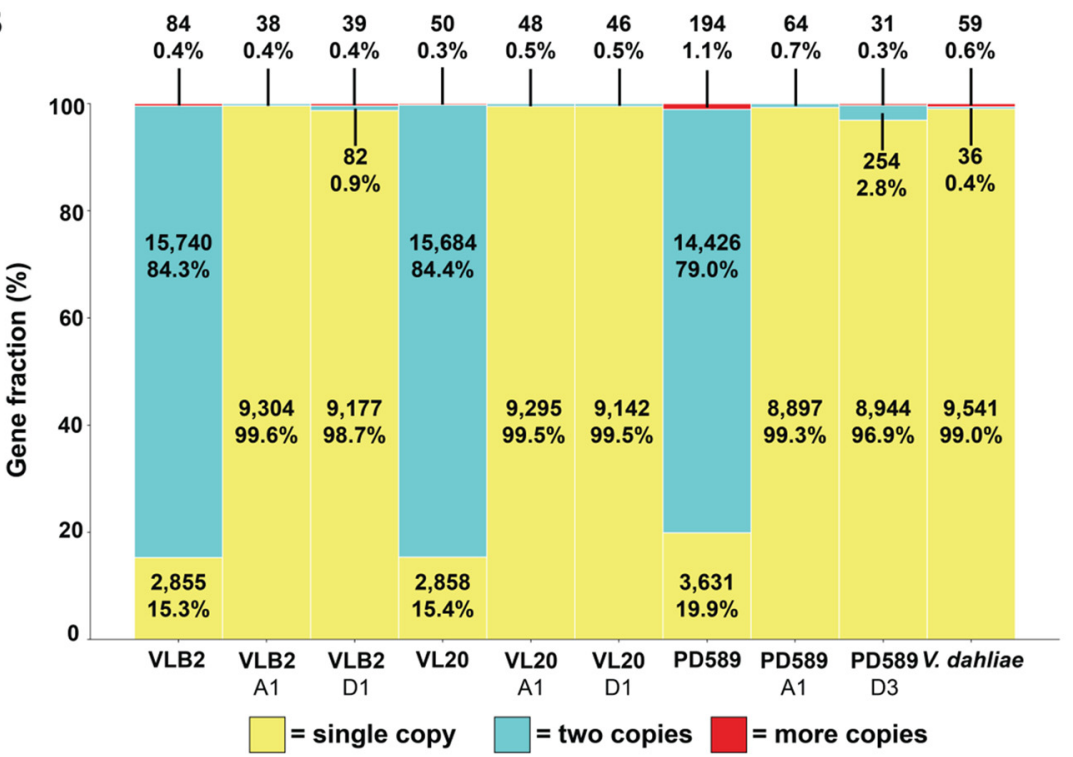

VL20

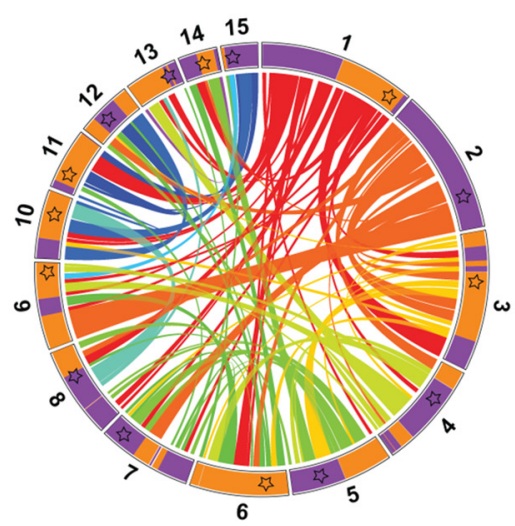

PD589

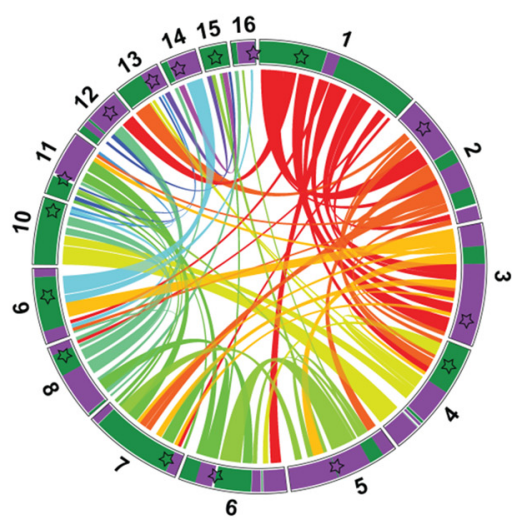

= A1 sub-genome

\section{= D3 sub-genome}

FIG 2 Verticillium longisporum genes with two copies are almost exclusively homeologs. (A) Schematic overview of different evolutionary origins of homologous genes in hybrids. Paralogs are homologous genes that originate from gene duplication, while orthologous genes originate by speciation. Homeologs are homologous genes originating from a hybridization event. (B) The gene fractions occurring in single, two, and more than two copies in $V$. longisporum strains VLB2, VL20, and PD589, with V. dahliae (strain JR2) as a comparison. "A1," "D1," and "D3" represent species A1, D1, and D3 subgenomes, respectively. (C) Homeologous regions within the $V$. longisporum genomes. Ribbons indicate homeologous regions within the strains, and contig colors indicate the parental origins, similar to Fig. 1.

breaks (Fig. 3B). Rearrangement occurred in the majority of the chromosomes as only 2 and 1 chromosomes did not have syntenic breaks in VLB2 and VL20, respectively (Fig. 3B). As genomic rearrangements are often associated with repeat-rich genome regions, such as in $V$. dahliae $(47,49,50)$, the synteny breakpoints were tested for their association with repetitive regions. Since the median repeat fraction in a 20-kb window around the repeats is $10.8 \%$, which is significantly higher than the median repeat fraction based on random sampling (average $=3.1 \% ; \sigma=0.78 \%$ ) (Fig. S6), it can be concluded that the chromosomal rearrangements are also similarly associated with repeats in V. longisporum. Furthermore, of the 46 breakpoints between VLB2 and VL20, 1 is associated with a VLB2 centromere, and 2 are associated with a VL20 centromere (Fig. 3B, red lines). In conclusion, chromosomal rearrangement rather than gene conversion is the main mechanism explaining the mosaic structure of the $V$. longisporum genome.

$V$. longisporum loses heterozygosity through deletions. To study putative gene losses in the aftermath of hybridization, we determined genes that have no homeolog 
A

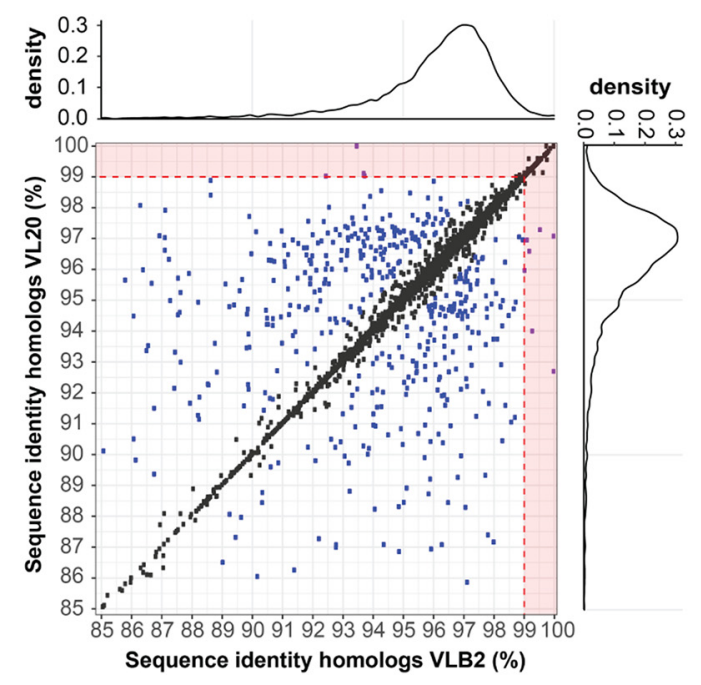

B

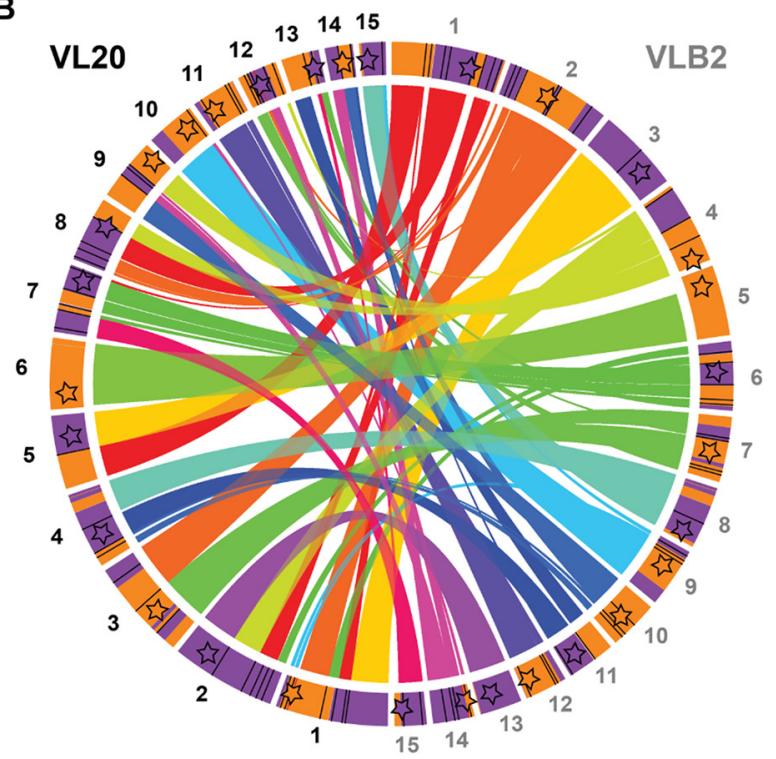

FIG 3 The mosaic genome structure of Verticillium longisporum originates from genomic rearrangements. (A) Contribution of gene conversion to $V$. longisporum genome evolution. Sequence identities between genes in copy, present in V. longisporum VLB2 and VL20, are depicted. Homologous gene pairs within a strain that encountered gene conversion are expected to have higher similarity within a strain than with the corresponding gene pair in the other strain. Gene pairs with a divergence of more than $1 \%$ in one $V$. longisporum strain and less than $1 \%$ in the other strain were considered conserved in the latter strain (purple dots in the red zones). In other cases, pairs that differ by less than $1 \%$ are depicted as a black dot, whereas a difference greater than $1 \%$ is depicted as a blue dot. (B) Contribution of genomic rearrangements to $V$. longisporum genome evolution. The $V$. longisporum chromosomes of strains VLB2 (right) and VL20 (left) are depicted. Ribbons indicate syntenic genome regions between the two strains, and contig colors indicate the parental origin, similar to Fig. 1 (purple, A1; orange, D1). Black lines on the chromosomes indicate synteny breaks that are not associated with centromeres, whereas red ones are associated with centromeres.

or paralog and can thus be considered to occur in a single copy. For the A1/D1 isolates, 15.3 to $15.4 \%$ of the genes occur in a single copy, whereas this fraction is $19.9 \%$ for $\mathrm{A} 1 /$ D3 isolate PD589 (Fig. 2B). We checked if proteins encoded by single-copy genes are enriched for particular Gene Ontology (GO) terms or Clusters of Orthologous Groups (COGs) or encode a protein with a signal peptide, which suggests that these proteins are secreted. No GO terms or COGs were enriched for the single-copy genes in any of the $V$. longisporum strains ( $P$ value of $<0.05$ by Fisher's exact test with BenjaminiHochberg correction). In total, 7.8 to $10.2 \%$ of the single-copy genes encode a protein with a signal peptide, which is significantly lower than the 11.9 to $12.3 \%$ for genes with a homologous copy in the same genome ( $P$ value of $<0.05$ by Fisher's exact test). Of the A1/D1 single-copy genes, $52 \%$ reside in the $A 1$ subgenome, and $47 \%$ reside in the D1 subgenome. Similarly, for PD589, 49\% and 50\% reside in the A1 and D3 subgenomes, respectively. Thus, single-copy genes are equally distributed across the two subgenomes in $V$. longisporum. Single-copy genes can originate from either gene loss or parent-specific contributions to the hybrid. Since VLB2 and VL20 originate from the same hybridization event (40), we can quantify how many single-copy genes originate from gene loss during the divergence of VLB2 and VL20. In total, 14.7 to $14.8 \%$ of the singly-copy genes have at least one copy in each subgenome of the other A1/D1 strain, suggesting that gene deletion is an ongoing process in $V$. longisporum evolution. Of the single-copy genes that lost their homeolog after the hybridization event, $48 \%$ resided in the species A1 subgenome, whereas 51 to $52 \%$ resided in the D1 subgenome, suggesting that gene losses occurred to similar extents in each of the subgenomes. Furthermore, 24\% (14 clusters) and 15\% (13 clusters) of genes in VLB2 and VL20, respectively, that are lost after the divergence of these two strains are clustered in the other strain, indicating that large deletions occur. The gene clusters lost in VL20 do not localize at any of the 46 synteny breakpoints between VLB2 and VL20 (Fig. 3B). In contrast, 4 of the 14 clusters that are lost in VLB2 are associated with such 

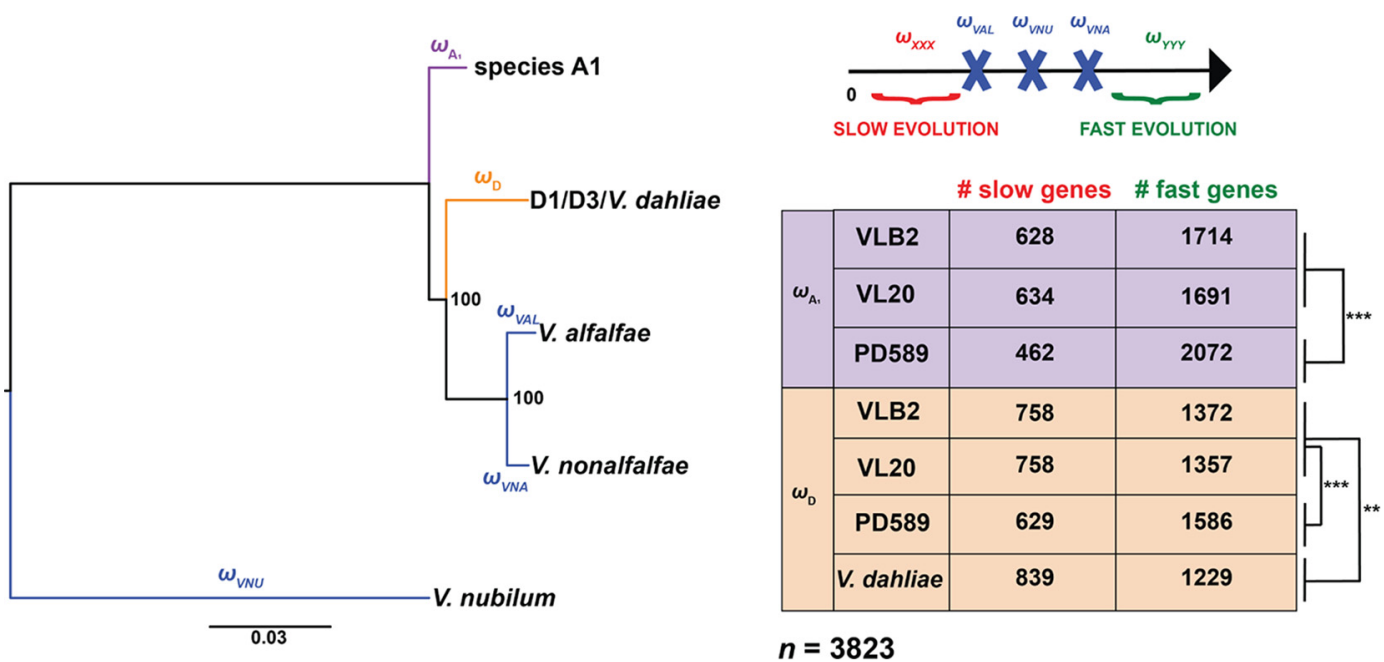

FIG 4 Verticillium longisporum genes diverge faster than Verticillium dahliae orthologs. $K_{a} / K_{\mathrm{s}}$ ratios $(\omega)$ were calculated for the tree branches leading to Verticillium spp. of the clade Flavnonexudans genomes and the $V$. longisporum subgenomes. A total of 3,823 genes with one ortholog in all respective Verticillium (sub)genomes were analyzed. $V$. longisporum and $V$. dahliae genes with fast or slow evolution have a higher $\omega$ or lower $\omega$, respectively, than their $V$. alfalfae (VAL) $V$. nonalfalfae (VNA), and V. nubilum (VNU) orthologs. Significance in gene numbers was calculated with Fisher's exact test. **, $P<0.01 ;{ }^{* * *}, P<0.001$.

breakpoints. Thus, genomic recombination may lead to the loss of gene clusters but does not explain the majority of the gene losses.

Acceleration of gene evolution upon hybridization. To investigate gene sequence evolution after hybridization, we compared the ratios of nonsynonymous $\left(K_{a}\right)$ and synonymous $\left(K_{s}\right)$ substitutions $(\omega)$ for branches leading to Verticillium species (Fig. 4). To exclude the putative impact of the (partial) chromosome 13 duplication in PD589, we excluded genes of this chromosome from the analysis. Substitution rates were determined for a total of 3,823 genes that have just one ortholog in the analyzed Verticillium species, $V$. alfalfae, $V$. dahliae, $V$. nonalfalfae, and $V$. nubilum, as well as in each of the $V$. longisporum subgenomes. To mitigate possible biases of different divergence times between the Verticillium species, we performed the analyses four times: three times with the two subgenomes of V. longisporum strains VLB2, VL20, and PD589 and once with $V$. dahliae and the A1 subgenome of VLB2 (Fig. 4). V. longisporum and $V$. dahliae genes with higher $\omega$ values than their $V$. alfalfae, $V$. nonalfalfae, and $V$. nubilum orthologs were considered quickly evolving, whereas those with lower $\omega$ values were considered slowly evolving. Comparing the D1/D3/V. dahliae branch, V. dahliae has 839 slowly evolving genes, which is a higher number than the 758 and 629 slowly evolving genes of the $V$. longisporum D1 and D3 subgenomes, respectively. Conversely, V. dahliae has 1,229 quickly evolving genes, which is lower than the number found for the $V$. longisporum D1 and D3 subgenomes, 1,357/1,372 (VL20/VLB2) and 1,586, respectively (Fig. 4). This observation fits the prevailing hypothesis that hybridization accompanied by genome duplication has a "relaxing" effect on gene evolution $(32,51)$. Furthermore, the lower number of slowly evolving genes and the higher number of quickly evolving genes in the D3 subgenome are significantly different from those of the D1 subgenome ( $P<0.001$ by Fisher's exact test). Similar to the D subgenomes, the A1 subgenome of lineage A1/D3 has a higher number of quickly evolving genes ( 2,072 versus 1,691 to 1,714 ) and a lower number of slowly evolving genes (462 versus 628 to 634) than the $A 1$ subgenome of lineage A1/D1. In conclusion, $V$. longisporum lineage A1/D3 genes generally evolve faster than lineage A1/D1 genes in both subgenomes. This may indicate that $A 1 / D 3$ evolved for a longer time under the more relaxed gene evolutionary conditions than A1/D1; i.e., A1 and D3 hybridized a longer time ago than A1/D1.

To see whether particular genes evolve faster, we functionally characterized the $V$. longisporum A1/D3 genes that have a higher $\omega$ value than their $V$. alfalfae, $V$. 
A

A1/D1 A1/D3

VLB2 VL20 PD589

\begin{tabular}{|c|c|c|c|c|c|c|c|}
\hline \multirow{3}{*}{$\begin{array}{l}421 \\
7.5 \%\end{array}$} & & \multirow{3}{*}{$\begin{array}{c}631 \\
11.3 \%\end{array}$} & \multirow{3}{*}{ One homeolog upregulated } & \multirow[b]{2}{*}{ م } & & & \\
\hline & 433 & & & & 156 & 167 & 253 \\
\hline & $7.7 \%$ & & & \multirow[b]{2}{*}{$a a_{0}$} & 265 & 266 & 378 \\
\hline \multirow{2}{*}{$\begin{array}{l}470 \\
8.4 \%\end{array}$} & 538 & \multirow{2}{*}{$\begin{array}{c}741 \\
13.2 \%\end{array}$} & \multirow{2}{*}{ One homeolog downregulated } & & 187 & 198 & 328 \\
\hline & $9.6 \%$ & & & & 283 & 340 & 413 \\
\hline \multirow{2}{*}{$\begin{array}{c}20 \\
0.4 \%\end{array}$} & 16 & 24 & \multirow{2}{*}{ Haploid intermediate expression } & & 10 & 8 & 13 \\
\hline & $0.3 \%$ & $0.4 \%$ & & & 10 & 8 & 11 \\
\hline 3764 & 3718 & 2919 & \multirow{2}{*}{ No differential expression } & \multirow{2}{*}{\multicolumn{2}{|c|}{$=\mathrm{A} 1$ parent }} & & \\
\hline $67.2 \%$ & $66.3 \%$ & $52.1 \%$ & & & & & \\
\hline 435 & 419 & 541 & \multirow{2}{*}{ Both homeologs upregulated } & \multicolumn{2}{|c|}{$=V \cdot$ dahliae } & & \\
\hline $7.8 \%$ & $7.5 \%$ & $9.7 \%$ & & \multirow{2}{*}{\multicolumn{2}{|c|}{$=\mathrm{D}$ parent }} & & \\
\hline 494 & 480 & 748 & \multirow{2}{*}{ Both homeologs downregulated } & & & & \\
\hline $8.8 \%$ & $8.6 \%$ & $13.3 \%$ & & \multicolumn{2}{|l|}{$n=5604$} & & \\
\hline
\end{tabular}

B

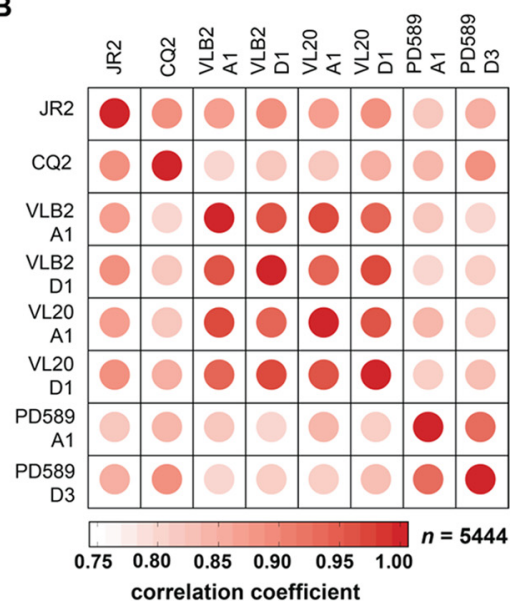

FIG 5 Gene expression patterns of Verticillium longisporum subgenomes display a remarkable resemblance. An expression pattern comparison between Verticillium longisporum subgenomes and Verticillium dahliae in culture medium was performed. (A) Differential expression between $V$. longisporum and $V$. dahliae genes. Only genes with one homolog in $V$. dahliae and two homeologs in $V$. longisporum strains VLB2, VL20, and PD589 were considered for differential expression. The significance of differential expression was calculated using $t$ tests relative to a threshold of a $\log _{2}$ fold change of 1 and a Benjamini-Hochberg-corrected $P$ value cutoff of 0.05 . (B) Expression pattern correlation between $V$. longisporum and $V$. dahliae. Only genes with one homolog in $V$. dahliae strains JR2 and CQ2 and two homeologs in V. longisporum strains VLB2, VL20, and PD589 were considered. Spearman's correlation coefficients ( $\rho$ ) were calculated based on the mean transcript-per-million values from three replicates.

nonalfalfae, and $V$. nubilum orthologs but also a higher value than their lineage A1/D1 homologs from the corresponding $\mathrm{A} 1$ and $\mathrm{D}$ subgenomes to select genes that quickly evolved after the A1 and D1/D3 last common ancestor. In total, 1,350 of the 3,823 (35.3\%) analyzed genes were quickly evolving in the PD589 A1 subgenome, and 1,084 (28.4\%) were quickly evolving in the D3 subgenome. We screened for GO term, COG, and secreted protein enrichments in these fast-evolving A1/D3 genes, and no enrichments for the COGs and genes encoding secreted proteins were found. In the A1 subgenome, $3 \mathrm{GO}$ terms with a molecular function were significantly enriched, associated with molecule binding (protein and ATP) and ATPase activity. In the D3 subgenome, "ATP binding" was the only significantly enriched GO term, which was also enriched in the A1 subgenome. In conclusion, the more pronounced "gene relaxation" in the A1/ D3 lineage than in the A1/D1 lineage does not clearly seem to affect genes with particular functions.

Expression pattern homogenization in the hybridization aftermath. To investigate the impact of hybridization on gene expression, the expression of $V$. longisporum genes was compared with that of $V$. dahliae orthologs from strains grown in vitro in potato dextrose broth (PDB). To this end, the expression of single-copy $V$. dahliae genes was compared with that of orthologs that are present in two homeologous copies in three V. longisporum strains (VLB2, VL20, and PD589). Genes on chromosome 13 from strain PD589 and their homologs were excluded from the analysis to avoid putative biases due to a (partial) chromosome duplication, and in total, 5,604 expressed genes were compared. RNA sequencing reads were mapped to the predicted $V$. longisporum genes, of which 50 to $51 \%$ mapped to species $A 1$ homeologs and 49 to $50 \%$ mapped to the $D$ homeologs. Thus, we observed no global differences in overall contributions to gene expression of the subgenomes. Over half of the $V$. longisporum homeologs display no differential expression with their $V$. dahliae orthologs, indicating that the majority of the genes did not evolve differential expression patterns (Fig. 5A). In both lineages, higher numbers of differently expressed genes were found in the A1 subgenome than in the D subgenomes: 27 versus $23 \%$ for $A 1 / D 1$ and 38 versus $34 \%$ for A1/D3, respectively. The higher fraction of differentially expressed A1 genes is in accordance with the more distant phylogenetic relationship of parent $\mathrm{A} 1$ with $V$. dahliae than of the D parents (Fig. S5). Intriguingly, although D3 diverged more recently from 
V. dahliae than D1, D3 has more differentially expressed orthologs with $V$. dahliae than D1. When comparing expression patterns between subgenomes, 11 to $13 \%$ of the genes display differential expression between their A1 and D homeologs. Intriguingly, this is more than half the number of differentially expressed $D$ and $V$. dahliae orthologs (23 to $34 \%$ ) despite the fact that the $D$ parents diverged more recently from $V$. dahliae than from species A1 (Fig. S5). In general, the gene expression patterns of the A1 and D subgenomes of the same hybridization event are highly correlated (0.93 to 0.96), higher than D subgenomes and $V$. dahliae strain JR2 (0.85 to 0.89) and higher than the A1 subgenomes between hybridization events ( 0.82 to 0.84 ) (Fig. 5B; Table S2). To compare these expression patterns with the gene expression variation between different $V$. dahliae strains, we sequenced RNA from cotton-infecting $V$. dahliae strain CQ2 grown in potato dextrose broth. Although JR2 and CQ2 belong to the same species, their overall gene expression patterns are more dissimilar $(\rho=0.89)$ than that of $V$. longisporum subgenomes (Fig. 5B; Table S2). The overall discrepancy in the phylogenetic relationship and expression pattern similarities suggests that the subgenome expression patterns of the subgenomes in $V$. longisporum homogenized upon hybridization.

Differential homeolog expression occurs in particular gene categories. Although parental gene expression patterns appear to have globally homogenized upon hybridization, differential homeolog expression occurs as well (Fig. 5). To assess if genes with differential homeolog expression belong to specific gene groups, we screened for functional enrichments. In total, 10\% of the fast-evolving PD589 genes (defined above) have differential homeolog expression, which is significantly lower than the $12 \%$ differential homeolog expression for the remainder of the genes $(P<0.05$ by Fisher's exact test). In both the A1/D1 and A1/D3 lineages, genes with differential homeolog expression are enriched for $\mathrm{GO}$ terms related to oxidation-reduction processes, transmembrane transport, and flavin adenine dinucleotide (FAD) binding (Fig. 6A; Table S3). Additionally, the COGs "carbohydrate transport and metabolism" and "secondary metabolite biosynthesis, transport, and catabolism" (Q) are enriched in both lineages (Table S3). Furthermore, we tested if genes encoding secreted proteins were significantly enriched among the genes with differential homeolog expression. Indeed, 23 and $16 \%$ of the genes with differential homeolog expression code for a secreted protein in the lineage A1/D1 isolates and the A1/D3 isolate, respectively, whereas this is $9 \%$ of the genes that do not display differential expression among homeologs (VLB2, $P=1.23 \mathrm{E}-32 ; \mathrm{VL20}, P=3.71 \mathrm{E}-29 ; \mathrm{PD} 589, P=1.14 \mathrm{E}-08$ [by Fisher's exact test]). In conclusion, differential homeolog expression seems to be important for particular gene categories, including categories that can be implicated in plant pathogenicity.

Homeolog-specific expression responses upon plant colonization. Considering the plant-pathogenic nature of $V$. longisporum and also that genes encoding secreted proteins, which are often implicated in pathogenicity on host plants, are enriched among the genes with differential homeolog expression, we assessed homeolog-specific gene expression during plant colonization. To this end, oilseed rape plants were inoculated with the $V$. longisporum strains VLB2, VL20, and PD589. As observed previously, oilseed rape plants inoculated with VLB2 and PD589 developed typical Verticillium symptoms, including stunted plant growth and leaf chlorosis (52). In contrast, oilseed rape plants inoculated with VL20 did not display any disease symptoms. Consequently, we performed total RNA sequencing for oilseed rape plants inoculated with V. longisporum strains VLB2 and PD589. For strain PD589, genes on chromosome 13 and their homeologs were removed from the analysis. To assess the quality of the RNA-Seq data, the expression of the 15 highest in planta-induced PD589 genes was verified by real-time PCR. In total, 14 of the 15 tested genes had, similar to the RNASeq data, a drastic increase in expression upon plant colonization, showing that the real-time PCR data generally correspond to the RNA-Seq data (Fig. S7). For VLB2 and PD589, 51\% of the reads mapped to the A1 subgenome, and 49\% mapped to the D subgenome. Thus, similar to in vitro-grown $V$. longisporum, we did not observe any global difference in the overall contributions to gene expression of one of the 


\section{Differential homeolog expression}

A

GO term enrichment for differential homeolog expression

\begin{tabular}{|c|c|c|c|c|c|}
\hline GO number & GO term & VLB2 & VL20 & PD589 & PD589 \\
\hline GO:0004497 & $\begin{array}{c}\text { monooxygenase } \\
\text { activity }\end{array}$ & 9 & 7 & & \\
\hline GO:0005506 & $\begin{array}{l}\text { iron ion } \\
\text { binding }\end{array}$ & 19 & 21 & & 6 \\
\hline GO:0008061 & $\begin{array}{c}\text { chitin } \\
\text { binding }\end{array}$ & & 5 & & \\
\hline GO:0008237 & $\begin{array}{l}\text { metallopeptidase } \\
\text { activity }\end{array}$ & 8 & 7 & & \\
\hline GO:0016491 & $\begin{array}{l}\text { oxidoreductase } \\
\text { activity (OA) }\end{array}$ & 67 & 64 & 59 & \\
\hline GO:0016614 & $\begin{array}{l}\mathrm{OA} \text { on } \mathrm{CH}-\mathrm{OH} \\
\text { group of donors }\end{array}$ & 6 & 8 & & \\
\hline GO:0016705 & $\begin{array}{c}\text { OA on paired donors } \\
\text { incorp/red mol oxygen }\end{array}$ & 19 & 20 & & 5 \\
\hline GO:0020037 & $\begin{array}{l}\text { heme } \\
\text { binding }\end{array}$ & 23 & 20 & & 7 \\
\hline GO:0022857 & $\begin{array}{l}\text { transmembrane } \\
\text { transporter activity }\end{array}$ & 64 & 56 & 47 & \\
\hline GO:0050660 & $\begin{array}{c}\text { flavin adenine } \\
\text { dinucleotide binding }\end{array}$ & 21 & 23 & & 9 \\
\hline GO:0071949 & $\begin{array}{c}\text { FAD } \\
\text { binding }\end{array}$ & 13 & 13 & 11 & 7 \\
\hline GO:0016020 & membrane & & & 42 & \\
\hline GO:0005975 & $\begin{array}{l}\text { carbohydrate } \\
\text { metabolic process }\end{array}$ & & 33 & & \\
\hline GO:0006725 & $\begin{array}{l}\text { aromatic compound } \\
\text { metabolic process }\end{array}$ & & 4 & & \\
\hline GO:0055085 & $\begin{array}{c}\text { transmembrane } \\
\text { transport }\end{array}$ & 70 & 64 & 60 & \\
\hline GO:0055114 & $\begin{array}{l}\text { oxidation-reduction } \\
\text { process }\end{array}$ & 101 & 92 & 72 & 25 \\
\hline \multicolumn{3}{|c|}{$=$ Molecular Function } & \multicolumn{3}{|c|}{$=$ culture medium } \\
\hline & \multicolumn{2}{|l|}{ = Cellular Component } & \multicolumn{3}{|c|}{$=$ in planta } \\
\hline
\end{tabular}

B

Homeologs with differential in planta expression

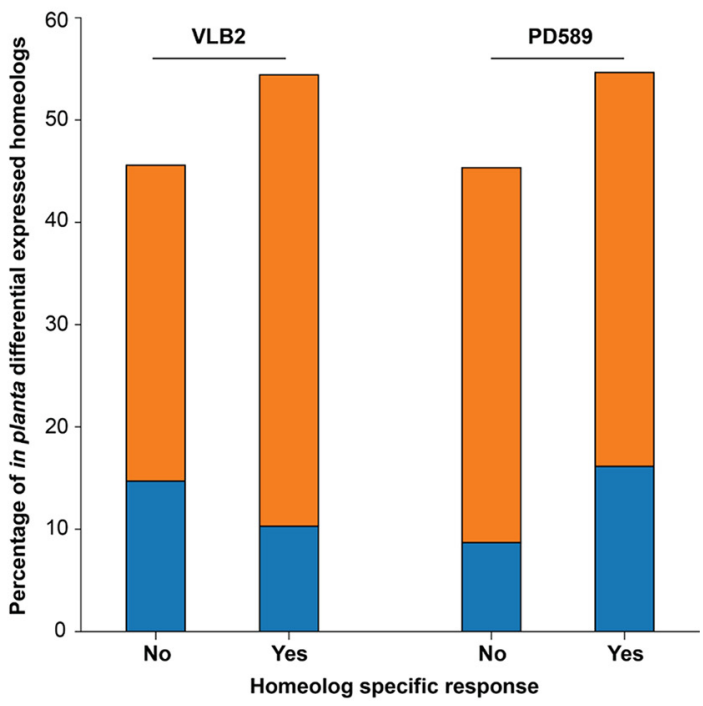

= signal peptide $\square=$ no signal peptide

FIG 6 Verticillium longisporum displays subgenome-specific gene expression responses. Functional enrichments for Verticillium longisporum genes with differential homeolog expression in culture medium and in planta are shown. Only $V$. longisporum genes with two homeologs were considered. (A) Gene Ontology (GO) terms that are significantly enriched in differentially expressed homeologs of VLB2, VL20, and PD589. A more detailed overview and levels of significance are reported in Table S3 in the supplemental material. The numbers of genes with differential homeolog expression are indicated. (B) Fractions of genes with differential homeolog expression in planta with and without a homeolog-specific response. Genes have a homeolog-specific response if they display differential homeolog expression in planta and have no differential or the opposite expression ratio for $V$. longisporum grown in culture medium.

subgenomes in planta. In total, $1.1 \%$ and $2.7 \%$ of the homeologs displayed differential expression in planta, which is lower than the 11.3 and $13.4 \%$ found for VLB2 and PD589 grown in vitro, respectively. Genes with differential homeolog expression in planta were not enriched for any GO term in the A1/D1 strain VLB2 (Table S3), whereas in the A1/D3 strain PD589, differentially expressed homeologs were enriched for GO terms associated with oxidation-reduction processes and molecular binding (iron ion, heme, and FAD) (Fig. 6A; Table S3). For A1/D1 and A1/D3, genes with differential homeolog expression were enriched for those encoding secreted proteins: $25 \%$ of the differentially expressed homeologs encode secreted proteins, and 8 to $9 \%$ of the nondifferentially expressed homeologs encode other proteins $(P<0.05$ by Fisher's exact test). Thus, similar to in vitro-grown $V$. longisporum, differential homeolog expression in planta is especially important for genes encoding secreted proteins. In $33 \%$ of these secretome genes with differential homeolog expression in planta, no Pfam domain could be annotated, which is a feature often observed for effector proteins as they are often examples of biological innovation (53). Of these genes that could be functionally annotated, a carbohydrate-active enzyme (CAZyme) function was annotated in $32 \%$ of 
the cases. The remaining part of the functionally annotated genes with differential homeolog expression included other enzymes such as proteases, lipases, carboxylesterases, and peroxidases. We compared genes with differential homeolog expression in planta and in vitro to assess potential correlations. Intriguingly, over half (54 to 55\%) of the differentially expressed homeologs in planta are not differentially expressed in culture medium or have the inverse expression pattern, e.g., $A 1>D$ in vitro and $A 1<D$ in planta (Fig. 6B). Thus, over half of the genes with differential homeolog expression in planta display a homeolog-specific response compared to in vitro growth. For VLB2, $19 \%$ of these genes with a homeolog-specific response encode secreted proteins, whereas $32 \%$ of genes with similar differential homeolog expression in planta and in vitro encode secreted proteins. The opposite pattern was observed for PD589, i.e., 30\% with a homeolog-specific response and $19 \%$ with similar differential homeolog expression in planta and in vitro. However, these differences were not significant $(P>0.05$ by Fisher's exact test). In conclusion, different growth conditions cause homeolog-specific changes in the majority of the $V$. longisporum genes with differential homeolog expression, which are enriched in genes that encode secreted proteins.

\section{DISCUSSION}

Hybridization is a powerful evolutionary mechanism that can lead to the emergence of new plant pathogens with distinct features compared with their parents $(8,23)$. Here, we reveal the transcriptomic plasticity of the hybrid pathogen $V$. longisporum and illustrate the parental allele-specific response to different environmental cues. Differentially expressed $V$. longisporum homeologs are enriched for genes encoding secreted proteins that generally act to facilitate environmental manipulation (54). Interestingly, over half of the differentially expressed homeolog genes in planta display different relative contributions in vitro. Thus, upon the environmental changes that are associated with different growth conditions, $V$. longisporum encounters subgenomespecific gene expression alterations, leading to differential homeolog expression. Although not previously reported for any other hybrid plant pathogen, subgenomespecific gene expression alterations have previously been reported to occur in the artificial yeast hybrid $S$. cerevisiae $\times$ Saccharomyces uvarum upon a temperature change (55). Genes with these subgenome-specific responses were involved in a variety of biological processes, including the trehalose metabolic process that is involved in thermotolerance. Thus, more generally, hybrid fungi, comprising natural as well as artificial hybrids, respond to environmental changes in an allele-specific manner, especially for genes that manipulate or mitigate environmental changes. Secretome genes with differential homeolog expression in planta often have an enzymatic function or lack an annotated Pfam domain, which is a feature often observed for effector proteins that act in pathogenicity (53). Thus, conceivably, homeolog-specific responses in planta occur in genes that are important for host colonization. Similarly, differential homeolog expression in the hybrid opportunistic human pathogen Candida orthopsilosis involves genes that are implicated in host interactions, related to superoxide dismutase activity and zinc metabolism (56).

Although differential homeolog expression occurs, the general tendency is that expression patterns between the $\mathrm{A} 1$ and $\mathrm{D}$ subgenomes homogenized upon hybridization (Fig. 5). Despite the absence of A1 and D1 species due to their enigmatic nature, we can conclude that parental gene expression patterns homogenized in the aftermath of hybridization as subgenome expression patterns display more resemblance than the expression pattern between $V$. dahliae and the $D$ parents and between the $A 1$ subgenomes of different hybridization events (Fig. 5B; see also Table S2 in the supplemental material). Homogenization of parental expression patterns has been similarly observed in the fungal allopolyploid Epichloë strain Lp1 (36) as well as in the artificial hybrid S. cerevisiae $\times$ S. uvarum, where the extent of differential ortholog expression between the parents was diminished upon hybridization (57). Thus, gene expression homogenization seems to be a more general phenomenon in fungi. Gene 
expression divergences may evolve through mutations in regulatory sequences of the gene itself (cis-effects), such as promoter elements, or alterations in other regulatory factors (trans-effects), such as chromatin regulation $(58,59)$. Conceivably, the higher correlation in homeolog expression patterns than in parental ortholog expression patterns originates from changes in trans regulators, as homeologs, in contrast to orthologs, share the same nuclear environment (59). Intriguingly, parent D3 has more genes that are differentially expressed with $V$. dahliae orthologs than parent D1, even though D3 diverged more recently from $V$. dahliae than D1 (Fig. 5; Fig. S5). Correspondingly, the A1 subgenome of lineage A1/D3 displays more differential gene expression with $V$. dahliae than the $A 1$ subgenome of the A1/D1 lineage. This may indicate that $A 1$ and D3 hybridized before $A 1$ and D1, as more distinct expression patterns may have evolved over time.

In addition to the transcriptomic plasticity of homeolog expression upon environmental changes, $V$. longisporum is also plastic on a genomic level, which is displayed by its mosaic structure (Fig. 1A; Table S1). Mosaicism is also observed in the grass pathogen Zymoseptoria pseudotritici, which is a close relative of the prominent wheat pathogen Zymoseptoria tritici (29). Z. pseudotritici is a homoploid hybrid that displays mosaicism on a population level where genome regions inherited from one parent display low variation, whereas highly variable genome regions were transmitted from both parents. $V$. longisporum mosaicism is caused by extensive genomic rearrangements after hybridization (Fig. 2B and Fig. 3). Genomic rearrangements are major drivers of evolution and facilitate adaptation to novel or changing environments (49). Genomic rearrangements are not specific to the hybrid nature of $V$. longisporum as other Verticillium species similarly encountered extensive chromosomal reshuffling (44, $45,50,60)$. In $V$. dahliae, genomic rearrangements especially occur in genomic regions that were originally described as lineage-specific regions, which are enriched for active transposable elements, and which are derived from segmental duplications that were followed by extensive reciprocal gene losses, encounter nucleotide sequence conservation, and have a unique epigenomic profile (50, 60-63). These lineage-specific regions are enriched for in planta-expressed genes and contain effector genes that facilitate host infection $(60,61,64,65)$. More recently, these lineage-specific regions have been referred to as dynamic chromosomal regions (61). Similar to $V$. dahliae, syntenic breaks in $V$. longisporum often reside in repeat-rich genome regions, as repetitive sequences (Fig. S6), due to their abundance, are more likely to act as a substrate for the unfaithful repair of double-strand DNA breaks $(49,50)$. However, the presence of two genomes within a single hybrid nucleus may also provide homeologous sequences with sufficient identity to mediate unfaithful repair.

The $V$. longisporum D genomes globally display accelerated evolution compared with their $V$. dahliae orthologs (Fig. 4), which may be a consequence of genome doubling. Interestingly, the $V$. longisporum A1/D3 lineage strain PD589 encountered more divergent gene evolution than the A1/D1 lineage strains VLB2 and VL20 in both subgenomes, indicating that the A1/D3 hybridization event occurred prior to the A1/D1 hybridization event as a longer allodiploid state could facilitate extended sequence divergence (66). However, accelerated evolution is not consistently observed in fungi as deceleration upon allopolyploidization has been recorded in the fungal genus Trichosporon (67). Arguably, environmental cues play an important role in the speed and grade of gene diversification upon allopolyploidization (68). Possibly, accelerated gene evolution in $V$. longisporum is cued by a host range alteration, as it is, in contrast to haploid Verticillium species, a Brassicaceae specialist (42). However, we did not find functional enrichments in fast-evolving genes that point toward that hypothesis. Moreover, as the A1 species remains enigmatic, we cannot be sure that a host shift occurred $(39,41)$.

Whole-genome duplication events are typically followed by extensive gene loss, often leading to reversion to the original ploidy state (69). For instance, the artificial interspecific hybrid S. cerevisiae $\times$ S. uvarum encountered nine independent events where 
loss of heterozygosity occurred after evolving for hundreds of generations under nutrient-limited conditions (70). Heterozygosity loss has proceeded to only a limited extent in V. longisporum, as $84 \%$ of lineage A1/D1 genes and 79\% of lineage A1/D3 genes are present in two copies, whereas the haploid $V$. dahliae contains only $0.4 \%$ of its genes in two copies (Fig. 2B). Thus, the $V$. longisporum genome displays the symptoms of a recent allodiploid, with gene loss being an ongoing process that by now has progressed only marginally. Heterozygosity loss can indicate deleterious epistatic interactions between parental genomes that need to homogenize in order for the hybrid to be viable. Similar to other fungal hybrids $(70,71)$, we did not observe a specific group of genes where loss of heterozygosity was selected for. The degree of haploidization is a third indication that the A1/D3 lineage likely hybridized prior to A1/D1, as haploidization progressed further in A1/D3 than in A1/D1 (Fig. 2B). C. orthopsilosis hybrids from different hybridization events have different degrees of heterozygosity loss, but genes where homeologs are maintained in both hybrids are enriched for those that have differential homeolog expression (56). Although species often revert to their original ploidy state after polyploidization, the retention of both homeolog copies can also be evolutionarily advantageous, for instance, to respond in a parental allele-specific fashion to environmental cues (Fig. 6).

Conclusion. Allodiploidization is an intrusive evolutionary mechanism in fungi where two chromosome sets from parents with distinct evolutionary histories merge. Consequently, most genes obtain an additional gene copy that can be differentially regulated according to the environmental conditions. Thus, allodiploid fungi can respond in a parental allele-specific fashion to environmental cues. Besides such parental allele-specific gene expression, allodiploidization furthermore contributed to dynamic genome evolution through rearrangements between parental chromosome sets and accelerated gene evolution in $V$. longisporum. Thus, in comparison to haploid Verticillium species, $V$. longisporum has high adaptive potential that can contribute to host immunity evasion and may explain its specialization toward brassicaceous plant hosts.

\section{MATERIALS AND METHODS}

V. longisporum genome sequencing and assembly. Genome assemblies of $V$. longisporum strains VLB2 and VL20 were previously constructed using long reads obtained through single-molecule realtime (SMRT) sequencing (40). Here, we sequenced V. longisporum strain PD589 using Oxford Nanopore technology. In order to obtain DNA of PD589, spores were harvested from potato dextrose agar (PDA) plates and grown in 1/5 potato dextrose broth (PDB) for 5 days. Mycelium and spores were collected on Microcloth, freeze-dried overnight, and ground to a fine powder. For DNA isolation, $100 \mathrm{mg}$ of material was used and incubated for $1 \mathrm{~h}$ at $65^{\circ} \mathrm{C}$ with $800 \mu$ l DNA extraction buffer $(0.35 \mathrm{M}$ sorbitol, $0.1 \mathrm{M}$ Tris base, $5 \mathrm{mM}$ EDTA [pH 7.5]), nucleic lysis buffer (0.2 M Tris, $0.05 \mathrm{M}$ EDTA, $2 \mathrm{M} \mathrm{NaCl}, 2 \%$ cetyltrimethylammonium bromide $[C T A B])$, and Sarkosyl (10\%, wt/vol) in a 2:2:1 ratio. Subsequently, a 1/2 volume of phenol-chloroform-isoamyl alcohol (25:24:1) was added, and the mixture was shaken vigorously and incubated at room temperature (RT) for $5 \mathrm{~min}$ before centrifugation at maximum speed (16,000 rpm) for 15 $\min (\mathrm{RT})$. The upper (aqueous phase) layer was transferred to a new tube, $5 \mu \mathrm{l}$ of RNase $(10 \mathrm{mg} / \mu \mathrm{l})$ was added, and the mixture was incubated at $37^{\circ} \mathrm{C}$ for $1 \mathrm{~h}$. Next, a $1 / 2$ volume of chloroform was added, mixed, and centrifuged at maximum speed for $10 \mathrm{~min}$ at RT. The upper layer was transferred to a new tube, and a second chloroform wash step was performed. After transferring the upper layer to a new tube, it was mixed with 1 volume $(\sim 800 \mu \mathrm{l})$ of $100 \%$ ice-cold ethanol by gently inverting the tube, and finally, the DNA was fished out and washed twice by applying $500 \mu \mathrm{l}$ of $70 \%$ ethanol. Finally, the DNA was air dried, resuspended in nuclease-free water, and stored at $4^{\circ} \mathrm{C}$ overnight. The DNA quality, size, and quantity were assessed by nanodrop, gel electrophoresis, and Qubit analyses, respectively.

To sequence $V$. longisporum strain PD589 DNA, a library was prepared according to the manufacturer's protocol provided by ONT (catalog number SQK-RAD004), with an initial amount of $\sim 400 \mathrm{ng}$ high-molecular-weight (HMW) DNA. The library was loaded onto an R9.4.1 flow cell, which ran for $24 \mathrm{~h}$ and yielded $\sim 7 \mathrm{~Gb}$ of data. ONT sequencing reads were base called using Guppy (version 3.1.5), using the high-accuracy base-calling algorithm. Subsequently, adapter sequences were identified and removed using Porechop (version 0.2.3; default settings), adapters at the end of the reads were trimmed, and reads with internal adapters were discarded. To be able to polish the genome assembly, we used the same HWA DNA isolated for ONT sequencing to generate $\sim 35$ million high-quality ( $>95 \%$; Phred score of 20 ) 150-bp paired-end reads ( $\sim 76 \times$ coverage) using the BGISeq platform (BGI Tech Solutions, Hong Kong, China).

The V. longisporum PD589 genome was de novo assembled using Canu (version 1.8; genomeSize $=70 \mathrm{~m}$, corOutCoverage $=100$, batOptions $=^{\prime}-d g 3-d b 3-d r 1$-ca $500-c p$ 50') (72). In total, 924,740 cleaned ONT reads 
were used for the de novo assembly, of which 743,753 were $>1 \mathrm{~kb}(\sim 88 \times$ coverage). The genome assembly was polished using two sequential rounds of Apollo (version 1.1) (73). To this end, the high-quality pairedend reads were mapped to the genome assembly using bwa (version 0.7.17-r1188; default settings) (74).

To improve the assemblies to (nearly) the chromosome level, chromatin conformation capture (Hi-C) followed by high-throughput sequencing was performed for VLB2, VL20, and PD589, using methods similar to the ones previously reported (44). For the three $V$. longisporum strains, 1 million spores were added to $400 \mathrm{ml}$ potato dextrose broth and incubated for 6 days at $22^{\circ} \mathrm{C}$ with continuous shaking at $120 \mathrm{rpm}$. A total of $300 \mathrm{mg}$ (fresh weight) mycelium was used as the input for generating $\mathrm{Hi}-\mathrm{C}$ sequencing libraries with the Proximo Hi-C kit (microbe) (Phase Genomics, Seattle, WA, USA), according to the manufacturer's instructions. Hi-C sequencing libraries were paired-end ( 2 by $150 \mathrm{bp}$ ) sequenced on the NextSeq500 platform at USEQ (Utrecht, The Netherlands). Juicer (v1.6) was then used to map Hi-C sequencing reads to the previously obtained assemblies (75). The contact matrices generated by Juicer were used by the three-dimensional (3D) de novo assembly (3D-DNA) pipeline (v180922) to eliminate misjoints in the previous assemblies (76). The assemblies were manually further improved using Juicebox Assembly Tools (JBAT) (v.1.11.08) (77). JBAT was subsequently used to determine the centromere location based on intra- and interchromosomal contact frequencies. Only contigs that were larger than $100 \mathrm{~kb}$ were maintained in the assembly. Coverage of ONT sequencing for the $V$. longisporum PD589 assembly was determined for 20-kb windows with SAMtools depth (v1.9) (78), and reads were mapped with minimap2 (v2.17-r941) (79).

The mitochondrial genomes of the haploid Verticillium species were previously sequenced and assembled (45). Mitochondrial $V$. longisporum genomes were assembled alongside the nuclear genomes (40). Mitochondrial contigs consisted of multiple copies of the mitochondrial genome due to its circular nature. A single copy of the mitochondrial genome was excised using BEDTools getfasta (v2.23.0) (102). Filtered $V$. longisporum subreads were mapped to these single-copy mitochondrial assemblies using circlator (v1.5.5) (103). The mapped reads were subsequently used to make a new V. longisporum mitochondrial genome assembly using SAMtools mpileup (v1.8) (78).

RNA sequencing. To obtain RNA-Seq data for Verticillium grown in culture medium, $V$. dahliae isolates JR2 and CQ2 and V. longisporum isolates VLB2, VL20, and PD589 were grown for 3 days in PDB, with three biological replicates for each isolate. To obtain RNA-Seq data from in planta growth, 2-weekold plants of the susceptible oilseed rape cultivar 'Quartz' were inoculated by dipping the roots for 10 $\mathrm{min}$ in a spore suspension of $1 \times 10^{6}$ conidiospores $\mathrm{ml}^{-1}$ of $\mathrm{V}$. longisporum isolates VLB2, VL20, and PD589 (52). After root inoculation, plants were grown in individual pots in a greenhouse under a cycle of $16 \mathrm{~h}$ of light and $8 \mathrm{~h}$ of darkness, with temperatures maintained between $20^{\circ} \mathrm{C}$ and $22^{\circ} \mathrm{C}$ during the day and at a minimum of $15^{\circ} \mathrm{C}$ overnight. Three pooled samples (10 plants per sample) of stem fragments $(3 \mathrm{~cm})$ were used for total RNA extraction. Total RNA was extracted based on TRIzol RNA extraction (104). cDNA synthesis, library preparation (TruSeq RNA-Seq short-insert library), and Illumina sequencing (single-end $50 \mathrm{bp}$ ) were performed at the Beijing Genome Institute (BGI) (Hong Kong, China).

The extracted RNA was reverse transcribed according to the instructions of the Moloney murine leukemia virus (M-MLV) reverse transcriptase protocol (Promega, Madison, WI, USA). The expression of the tested genes was quantified with real-time PCR relative to VdGAPDH as previously described (64). The primers used can be found in Fig. S7. The assay was performed for three biological replicates of $V$. longisporum PD589 grown in planta and in vitro. The $\log _{2}$ fold change in gene expression was determined based on the median of the in planta and in vitro expression levels.

Gene prediction and functional characterization. The $V$. longisporum assemblies of strains VLB2, VL20, and PD589 and the previously published assemblies of $V$. dahliae strains JR2 and CQ2 $(47,62)$ were annotated using the BRAKER v2.1.4 pipeline for RNA-Seq data with the options "-softmasking" and "-fungus" enabled (48). RNA-Seq reads from Verticillium grown in axenic cultures (all replicates) were mapped to the assemblies using TopHat v2.1.1 (80). Predicted genes with internal stop codons, without a start codon, or with an unknown amino acid in the encoded protein sequence were removed from the analysis. The secretome prediction was done using SignalP5 (v5.0) (81). Pfam and Gene Ontology (GO) function domains were predicted using InterProScan (v5.42-78.0) (82). Clusters of Orthologous Group (COG) categories were determined for protein sequences using eggNOG-mapper (v2.0) with the taxonomic scope set on Ascomycota $(83,84)$. Carbohydrate-active enzymes (CAZymes) were annotated using the dbCAN2 meta server $(85,86)$. A protein was considered a CAZyme if at least two of the three tools (HMMER, DIAMOND, and Hotpep) predicted a CAZyme function.

Parental origin determination. Subgenomes were divided based on the differences in sequence identities between species A1 and D1/D3 with V. dahliae. V. longisporum genomes of VLB2, VL20, and PD589 were aligned to the complete genome assembly of $V$. dahliae JR2 using NUCmer (v3.1), which is part of the MUMmer package (87). Here, only 1-to-1 alignments longer than $10 \mathrm{~kb}$ and with a minimum of $80 \%$ identity were retained. Subsequent alignments were concatenated if they aligned to the same contig with the same orientation and order as the reference genome so that lineage-specific genomic regions could also be assigned to a subgenome. The average nucleotide identity was determined for every concatenated alignment and used to divide the genomes into subgenomes. In addition, the sequence identities of orthologous coding regions between $V$. longisporum and $V$. dahliae were determined using the Needle-Wunsch algorithm implemented in EMBOSS (v6.6.0.0) (88). Alignment and coding sequence identities were both used to determine the parental origin of genome regions. Differences in GC content between homologous genes present in two copies were calculated as described previously (28). GC contents of gene coding regions were calculated with infoseq from EMBOSS (v.6.6.0.0) (88). The features to indicate the biparental origin of the $V$. longisporum genomes were visualized using the R package circlize (v.0.4.10) (89). 
Genome analysis. The quality of genome assemblies was assessed by screening for the presence of benchmarking universal single-copy orthologs (BUSCOs) using BUSCO software version 4.0.6 with the database "ascomycota_odb10" (90).

Repeats were de novo identified using RepeatModeler (v1.0.11) and combined with the repeat library from RepBase (release 20170127) (91). The genomic location of repeats was identified with RepeatMasker (v4.0.6).

The phylogenetic relationship of the nuclear and mitochondrial (sub)genomes of the Verticillium species of the clade Flavnonexudans (38) was determined using the following haploid strains: $V$. alfalfae PD683, V. dahliae JR2, V. nonalfalfae TAB2, and V. nubilum PD621 $(45,47)$. Phylogenetic trees based on nuclear DNA were constructed based on the ascomycete BUSCOs that were shared by all the included species (90). Nucleotide sequences were separately aligned using MAFFT (v7.464) (92). Phylogenetic trees were inferred using RAxML with the Generalised Time Reversible (GTR) + GAMMA substitution model (v8.2.11) (93). The robustness of the inferred phylogeny was assessed by 100 rapid bootstrap approximations.

Homologs in Verticillium were determined using nucleotide BLAST (v2.2.31+). Genes with a minimum identity of $80 \%$ and a minimum overlap of $80 \%$ were considered homologs, which were determined using SiLiX (v.1.2.10-p1) software (94).

Global nucleotide alignments using the Needle-Wunsch algorithm of the EMBOSS package were used to determine homologous gene pairs in VLB2 and VL20 (v6.6.0.0) (88). Sequence identities between these genes in copy were determined based on their global alignment. Synteny between the VLB2 and VL20 genome assemblies was determined by using one-to-one alignments obtained with NUCmer (v3.1), which is part of the MUMmer package (87). Synteny was visualized with the R package circlize (v.0.4.10) (89).

Gene clusters were identified using CROC with a minimum number of 3 genes that can be found for a cluster using a gene window of 6 (95). Here, the Benjamini-Hochberg method was used to correct for multiple testing.

Gene divergence. Previously published annotations of the haploid Verticillium species V. dahliae, $V$. alfalfae, $V$. nonalfalfae, $V$. nubilum, $V$. tricorpus, and $V$. albo-atrum were used to compare the evolutionary speeds of orthologs $(45,47)$. VESPA ( $11.0 \mathrm{~b})$ software was used to automate this process $(96)$. The coding sequences for each Verticillium species were filtered and subsequently translated using the VESPA "clean" and "translate" functions. Homologous genes were retrieved by protein BLAST (v2.2.31+) querying a database consisting of all Verticillium protein sequences. Here, the options "-max_hsps 1" and "-qcov_hsp_perc 80" were used. Homologous genes were grouped with the VESPA "best_reciprocal_group" function. Only homology groups that comprised a single representative for every Verticillium species were used for further analysis. Protein sequences of each homology group were aligned with muscle (v3.8) (97). The aligned protein sequences of the homology groups were converted to nucleotide sequences by the VESPA "map_alignments" function. The alignments were used to calculate the $K_{a} / K_{s}$ ratio for every branch of the species phylogeny using the codeml module of PAML (v4.9) with the following parameters: F3X4 codon frequency model, wag.dat empirical amino acid substitution model, and no molecular clock (98). To this end, the following phylogenetic tree topology was used: ((( V. dahliae/D1/ D3,(V. alfalfae, V. nonalfalfae)),A1),V. nubilum),(V. tricorpus, V. albo-atrum)). Divergence was compared only for genes that are present in the two subgenomes of V. longisporum strains VLB2, VL20, and PD589.

Gene expression analysis. The RNA sequencing reads were filtered using the Trinity software (v2.9.1) option Trimmomatic under standard settings (99). The reads were then mapped to the Verticillium genomes using Bowtie 2 (v2.3.5.1), with the first 15 nucleotides on the $5^{\prime}$ end of the reads being trimmed because of inferior quality (100). To compare gene expression patterns, homologs were retrieved by nucleotide BLAST (v2.2.31+). Genes with a minimum identity of $80 \%$ and a minimum overlap of $80 \%$ were considered homologs, which were determined using SiLiX (v.1.2.10-p1) software (94). Reads were counted for the predicted gene coding regions using the R package Rsubread (v1.34.7). Significant differential expression of a locus was calculated using the R package edgeR (v3.26.8) (101). The significance of differential expression was calculated using $t$ tests relative to a threshold of a $\log _{2}$ fold change of 1 with Benjamini-Hochberg correction using a $P$ value cutoff of 0.05 .

Data availability. Raw RNA-Seq reads and genome assemblies have been deposited at the NCBI database under the BioProject accession number PRJNA473305.

\section{SUPPLEMENTAL MATERIAL}

Supplemental material is available online only.

FIG S1, PDF file, 0.9 MB.

FIG S2, PDF file, $0.3 \mathrm{MB}$.

FIG S3, PDF file, $0.5 \mathrm{MB}$.

FIG S4, PDF file, 1.4 MB.

FIG S5, PDF file, $0.2 \mathrm{MB}$.

FIG S6, PDF file, $0.1 \mathrm{MB}$.

FIG S7, PDF file, 0.6 MB.

TABLE S1, DOC file, $0.05 \mathrm{MB}$.

TABLE S2, DOC file, $0.04 \mathrm{MB}$.

TABLE S3, DOC file, $0.1 \mathrm{MB}$. 


\section{ACKNOWLEDGMENTS}

We thank the Marie Curie Actions program of the European Commission that financially supported the research of J.R.L.D. Work in the laboratories of B.P.H.J.T. and M. F.S. is supported by the Research Council Earth and Life Sciences (ALW) of The Netherlands Organization of Scientific Research (NWO). B.P.H.J.T. acknowledges funding by the Alexander von Humboldt Foundation in the framework of an Alexander von Humboldt professorship endowed by the German Federal Ministry of Education and acknowledges support from the Deutsche Forschungsgemeinschaft (DFG) (German Research Foundation) under Germany's Excellence Strategy EXC 2048/1 (project identifier 390686111). The funders had no role in study design, data collection and analysis, decision to publish, or preparation of the manuscript.

We thank Sander Y. A. Rodenburg for sharing bioinformatics scripts.

\section{REFERENCES}

1. Barton NH. 2001. The role of hybridization in evolution. Mol Ecol 10:551-568. https://doi.org/10.1046/j.1365-294x.2001.01216.x.

2. Maheshwari S, Barbash DA. 2011. The genetics of hybrid incompatibilities. Annu Rev Genet 45:331-355. https://doi.org/10.1146/annurev-genet $-110410-132514$.

3. Blanckaert A, Bank C. 2018. In search of the Goldilocks zone for hybrid speciation. PLoS Genet 14:e1007613. https://doi.org/10.1371/journal .pgen.1007613.

4. Mallet J. 2007. Hybrid speciation. Nature 446:279-283. https://doi.org/10 $.1038 /$ nature05706.

5. Taylor SA, Larson EL. 2019. Insights from genomes into the evolutionary importance and prevalence of hybridization in nature. Nat Ecol Evol 3:170-177. https://doi.org/10.1038/s41559-018-0777-y.

6. Green RE, Krause J, Briggs AW, Maricic T, Stenzel U, Kircher M, Patterson N, Li H, Zhai W, Fritz MH-Y, Hansen NF, Durand EY, Malaspinas A-S, Jensen JD, Marques-Bonet T, Alkan C, Prüfer K, Meyer M, Burbano HA, Good JM, Schultz R, Aximu-Petri A, Butthof A, Höber B, Höffner B, Siegemund M, Weihmann A, Nusbaum C, Lander ES, Russ C, Novod N, Affourtit J, Egholm M, Verna C, Rudan P, Brajkovic D, Kucan Ž, Gušic I, Doronichev VB, Golovanova LV, Lalueza-Fox C, de la Rasilla M, Fortea J, Rosas A, Schmitz RW, Johnson PLF, Eichler EE, Falush D, Birney E, Mullikin JC, et al. 2010. A draft sequence of the Neandertal genome. Science 328:710-722. https://doi.org/10.1126/science.1188021.

7. Stukenbrock EH. 2016. The role of hybridization in the evolution and emergence of new fungal plant pathogens. Phytopathology 106:104-112. https://doi.org/10.1094/PHYTO-08-15-0184-RWW.

8. Depotter JRL, Seidl MF, Wood TA, Thomma BPHJ. 2016. Interspecific hybridization impacts host range and pathogenicity of filamentous microbes. Curr Opin Microbiol 32:7-13. https://doi.org/10.1016/j.mib.2016 .04 .005 .

9. Gabaldón T. 2020. Hybridization and the origin of new yeast lineages. FEMS Yeast Res 20:foaa040. https://doi.org/10.1093/femsyr/foaa040.

10. Leducq J-B, Nielly-Thibault L, Charron G, Eberlein C, Verta J-P, Samani P, Sylvester K, Hittinger CT, Bell G, Landry CR. 2016. Speciation driven by hybridization and chromosomal plasticity in a wild yeast. Nat Microbiol 1:15003. https://doi.org/10.1038/nmicrobiol.2015.3.

11. Marcet-Houben M, Gabaldón T. 2015. Beyond the whole-genome duplication: phylogenetic evidence for an ancient interspecies hybridization in the baker's yeast lineage. PLoS Biol 13:e1002220. https://doi.org/10 .1371/journal.pbio.1002220.

12. Mixão V, Gabaldón T. 2020. Genomic evidence for a hybrid origin of the yeast opportunistic pathogen Candida albicans. BMC Biol 18:48. https:// doi.org/10.1186/s12915-020-00776-6.

13. Mixão V, Hansen AP, Saus E, Boekhout T, Lass-Florl C, Gabaldón T. 2019. Whole-genome sequencing of the opportunistic yeast pathogen Candida inconspicua uncovers its hybrid origin. Front Genet 10:383. https:// doi.org/10.3389/fgene.2019.00383.

14. Pryszcz LP, Németh T, Saus E, Ksiezopolska E, Hegedüsová E, Nosek J, Wolfe KH, Gacser A, Gabaldón T. 2015. The genomic aftermath of hybridization in the opportunistic pathogen Candida metapsilosis. PLoS Genet 11:e1005626. https://doi.org/10.1371/journal.pgen.1005626.

15. Schröder MS, Martinez de San Vicente K, Prandini THR, Hammel S, Higgins DG, Bagagli E, Wolfe KH, Butler G. 2016. Multiple origins of the pathogenic yeast Candida orthopsilosis by separate hybridizations between two parental species. PLoS Genet 12:e1006404. https://doi.org/ 10.1371/journal.pgen.1006404.

16. Cook DE, Mesarich $\mathrm{CH}$, Thomma BPHJ. 2015. Understanding plant immunity as a surveillance system to detect invasion. Annu Rev Phytopathol 53:541-563. https://doi.org/10.1146/annurev-phyto-080614-120114.

17. Lo Presti L, Lanver D, Schweizer G, Tanaka S, Liang L, Tollot M, Zuccaro A, Reissmann S, Kahmann R. 2015. Fungal effectors and plant susceptibility. Annu Rev Plant Biol 66:513-545. https://doi.org/10.1146/annurev-arplant -043014-114623.

18. Rovenich H, Boshoven JC, Thomma BPHJ. 2014. Filamentous pathogen effector functions: of pathogens, hosts and microbiomes. Curr Opin Plant Biol 20:96-103. https://doi.org/10.1016/j.pbi.2014.05.001.

19. Snelders NC, Kettles GJ, Rudd JJ, Thomma BPHJ. 2018. Plant pathogen effector proteins as manipulators of host microbiomes? Mol Plant Pathol 19:257-259. https://doi.org/10.1111/mpp.12628.

20. Snelders NC, Rovenich H, Petti GC, Rocafort M, van den Berg GCM, Vorholt JA, Mesters JR, Seidl MF, Nijland R, Thomma BPHJ. 2020. Microbiome manipulation by a soil-borne fungal plant pathogen using effector proteins. Nat Plants 6:1365-1374. https://doi.org/10.1038/s41477 $-020-00799-5$.

21. Li F, Upadhyaya NM, Sperschneider J, Matny O, Nguyen-Phuc H, Mago R, Raley C, Miller ME, Silverstein KAT, Henningsen E, Hirsch CD, Visser B, Pretorius ZA, Steffenson BJ, Schwessinger B, Dodds PN, Figueroa M. 2019. Emergence of the Ug99 lineage of the wheat stem rust pathogen through somatic hybridisation. Nat Commun 10:5068. https://doi.org/10 .1038/s41467-019-12927-7.

22. Singh RP, Hodson DP, Jin Y, Lagudah ES, Ayliffe MA, Bhavani S, Rouse MN, Pretorius ZA, Szabo L, Huerta-Espino J, Basnet BR, Lan C, Hovmøller MS. 2015. Emergence and spread of new races of wheat stem rust fungus: continued threat to food security and prospects of genetic control. Phytopathology 105:872-884. https://doi.org/10.1094/PHYTO-01-15-0030-FI.

23. Menardo F, Praz CR, Wyder S, Ben-David R, Bourras S, Matsumae $H$, McNally KE, Parlange F, Riba A, Roffler S, Schaefer LK, Shimizu KK, Valenti $\mathrm{L}$, Zbinden H, Wicker T, Keller B. 2016. Hybridization of powdery mildew strains gives raise to pathogens on novel agricultural crop species. Nat Genet 48:201-205. https://doi.org/10.1038/ng.3485.

24. Runemark A, Vallejo-Marin M, Meier Jl. 2019. Eukaryote hybrid genomes. PLoS Genet 15:e1008404. https://doi.org/10.1371/journal.pgen.1008404.

25. McClintock B. 1984. The significance of responses of the genome to challenge. Science 226:792-801. https://doi.org/10.1126/science.15739260.

26. Matute DR, Butler IA, Turissini DA, Coyne JA. 2010. A test of the snowball theory for the rate of evolution of hybrid incompatibilities. Science 329:1518-1521. https://doi.org/10.1126/science.1193440.

27. Mixão V, Gabaldón T. 2018. Hybridization and emergence of virulence in opportunistic human yeast pathogens. Yeast 35:5-20. https://doi.org/10 .1002/yea.3242.

28. Louis VL, Despons L, Friedrich A, Martin T, Durrens $P$, Casarégola $S$, Neuvéglise C, Fairhead C, Marck C, Cruz JA, Straub M-L, Kugler V, Sacerdot C, Uzunov Z, Thierry A, Weiss S, Bleykasten C, De Montigny J, Jacques N, Jung P, Lemaire M, Mallet S, Morel G, Richard G-F, Sarkar A, Savel G, Schacherer J, Seret M-L, Talla E, Samson G, Jubin C, Poulain J, Vacherie B, Barbe V, Pelletier E, Sherman DJ, Westhof E, Weissenbach J, Baret PV, Wincker P, Gaillardin C, Dujon B, Souciet J-L. 2012. Pichia sorbitophila, an interspecies yeast hybrid, reveals early steps of genome 
resolution after polyploidization. G3 (Bethesda) 2:299-311. https://doi .org/10.1534/g3.111.000745.

29. Stukenbrock EH, Christiansen FB, Hansen TT, Dutheil JY, Schierup MH. 2012. Fusion of two divergent fungal individuals led to the recent emergence of a unique widespread pathogen species. Proc Natl Acad Sci U S A 109:10954-10959. https://doi.org/10.1073/pnas.1201403109.

30. Ortiz-Merino RA, Kuanyshev N, Braun-Galleani S, Byrne KP, Porro D, Branduardi $\mathrm{P}$, Wolfe $\mathrm{KH}$. 2017. Evolutionary restoration of fertility in an interspecies hybrid yeast, by whole-genome duplication after a failed mating-type switch. PLoS Biol 15:e2002128. https://doi.org/10.1371/ journal.pbio.2002128.

31. Van de Peer Y, Mizrachi E, Marchal K. 2017. The evolutionary significance of polyploidy. Nat Rev Genet 18:411-424. https://doi.org/10.1038/nrg .2017.26.

32. Hellsten U, Khokha MK, Grammer TC, Harland RM, Richardson P, Rokhsar DS. 2007. Accelerated gene evolution and subfunctionalization in the pseudotetraploid frog Xenopus laevis. BMC Biol 5:31. https://doi.org/10 .1186/1741-7007-5-31.

33. Grover CE, Gallagher JP, Szadkowski EP, Yoo MJ, Flagel LE, Wendel JF. 2012. Homoeolog expression bias and expression level dominance in allopolyploids. New Phytol 196:966-971. https://doi.org/10.1111/j.1469 -8137.2012.04365.x.

34. Zhang M, Tang YW, Qi J, Liu XK, Yan DF, Zhong NS, Tao NQ, Gao JY, Wang YG, Song ZP, Yang J, Zhang WJ. 2019. Effects of parental genetic divergence on gene expression patterns in interspecific hybrids of Camellia. BMC Genomics 20:828. https://doi.org/10.1186/s12864-019-6222-z.

35. Yoo M-J, Szadkowski E, Wendel JF. 2013. Homoeolog expression bias and expression level dominance in allopolyploid cotton. Heredity (Edinb) 110:171-180. https://doi.org/10.1038/hdy.2012.94.

36. Cox MP, Dong T, Shen G, Dalvi Y, Scott DB, Ganley ARD. 2014. An interspecific fungal hybrid reveals cross-kingdom rules for allopolyploid gene expression patterns. PLoS Genet 10:e1004180. https://doi.org/10.1371/ journal.pgen.1004180.

37. Praz CR, Menardo F, Robinson MD, Müller MC, Wicker T, Bourras S, Keller B. 2018. Non-parent of origin expression of numerous effector genes indicates a role of gene regulation in host adaption of the hybrid triticale powdery mildew pathogen. Front Plant Sci 9:49. https://doi.org/10.3389/ fpls.2018.00049.

38. Inderbitzin P, Bostock RM, Davis RM, Usami T, Platt HW, Subbarao KV. 2011. Phylogenetics and taxonomy of the fungal vascular wilt pathogen Verticillium, with the descriptions of five new species. PLoS One 6:e28341. https:// doi.org/10.1371/journal.pone.0028341.

39. Inderbitzin P, Davis RM, Bostock RM, Subbarao KV. 2011. The ascomycete Verticillium longisporum is a hybrid and a plant pathogen with an expanded host range. PLoS One 6:e18260. https://doi.org/10.1371/ journal.pone.0018260.

40. Depotter JRL, Seidl MF, van den Berg GCM, Thomma BPHJ, Wood TA. 2017. A distinct and genetically diverse lineage of the hybrid fungal pathogen Verticillium longisporum population causes stem striping in British oilseed rape. Environ Microbiol 19:3997-4009. https://doi.org/10 .1111/1462-2920.13801.

41. Depotter JR, Deketelaere $S$, Inderbitzin $P$, Tiedemann AV, Höfte $M$, Subbarao KV, Wood TA, Thomma BPHJ. 2016. Verticillium longisporum, the invisible threat to oilseed rape and other brassicaceous plant hosts. Mol Plant Pathol 17:1004-1016. https://doi.org/10.1111/mpp.12350.

42. Inderbitzin P, Subbarao KV. 2014. Verticillium systematics and evolution: how confusion impedes verticillium wilt management and how to resolve it. Phytopathology 104:564-574. https://doi.org/10.1094/PHYTO -11-13-0315-IA.

43. Varoquaux N, Liachko I, Ay F, Burton JN, Shendure J, Dunham MJ, Vert JP, Noble WS. 2015. Accurate identification of centromere locations in yeast genomes using Hi-C. Nucleic Acids Res 43:5331-5339. https://doi .org/10.1093/nar/gkv424.

44. Seidl MF, Kramer HM, Cook DE, Fiorin GL, van den Berg GCM, Faino L, Thomma BPHJ. 2020. Repetitive elements contribute to the diversity and evolution of centromeres in the fungal genus Verticillium. mBio 11: e01714-20. https://doi.org/10.1128/mBio.01714-20.

45. Shi-Kunne X, Faino L, van den Berg GCM, Thomma BPHJ, Seidl MF. 2018. Evolution within the fungal genus Verticillium is characterized by chromosomal rearrangements and gene losses. Environ Microbiol 20:1362-1373. https://doi.org/10.1111/1462-2920.14037.

46. Wang L, Sun Y, Sun X, Yu L, Xue L, He Z, Huang J, Tian D, Hurst LD, Yang S. 2020. Repeat-induced point mutation in Neurospora crassa causes the highest known mutation rate and mutational burden of any cellular life. Genome Biol 21:142. https://doi.org/10.1186/s13059-020-02060-w.

47. Faino $L$, Seidl M, Datema E, van den Berg GCM, Janssen A, Wittenberg AHJ, Thomma BPHJ. 2015. Single-molecule real-time sequencing combined with optical mapping yields completely finished fungal genome. mBio 6:e00936-15. https://doi.org/10.1128/mBio.00936-15.

48. Hoff KJ, Lomsadze A, Borodovsky M, Stanke M. 2019. Whole-genome annotation with BRAKER. Methods Mol Biol 1962:65-95. https://doi.org/ 10.1007/978-1-4939-9173-0_5.

49. Seidl MF, Thomma BPHJ. 2014. Sex or no sex: evolutionary adaptation occurs regardless. Bioessays 36:335-345. https://doi.org/10.1002/bies .201300155 .

50. Faino L, Seidl MF, Shi-Kunne X, Pauper M, van den Berg GCM, Wittenberg AHJ, Thomma BPHJ. 2016. Transposons passively and actively contribute to evolution of the two-speed genome of a fungal pathogen. Genome Res 26:1091-1100. https://doi.org/10.1101/gr.204974.116.

51. Kimura M, Ohta T. 1974. On some principles governing molecular evolution. Proc Natl Acad Sci U S A 71:2848-2852. https://doi.org/10.1073/ pnas.71.7.2848.

52. Depotter JRL, Rodriguez-Moreno L, Thomma BPHJ, Wood TA. 2017. The emerging British Verticillium longisporum population consists of aggressive Brassica pathogens. Phytopathology 107:1399-1405. https://doi .org/10.1094/PHYTO-05-17-0184-R.

53. Lanver D, Tollot M, Schweizer G, Lo Presti L, Reissmann S, Ma LS, Schuster M, Tanaka S, Liang L, Ludwig N, Kahmann R. 2017. Ustilago maydis effectors and their impact on virulence. Nat Rev Microbiol 15:409-421. https://doi.org/10.1038/nrmicro.2017.33.

54. Gagic D, Ciric M, Wen WX, Ng F, Rakonjac J. 2016. Exploring the secretomes of microbes and microbial communities using filamentous phage display. Front Microbiol 7:429. https://doi.org/10.3389/fmicb.2016.00429.

55. Li XC, Fay JC. 2017. cis-regulatory divergence in gene expression between two thermally divergent yeast species. Genome Biol Evol 9:1120-1129. https://doi.org/10.1093/gbe/evx072.

56. Hovhannisyan H, Saus E, Ksiezopolska E, Gabaldón T. 2020. The transcriptional aftermath in two independently formed hybrids of the opportunistic pathogen Candida orthopsilosis. mSphere 5:e00282-20. https://doi .org/10.1128/mSphere.00282-20.

57. Hovhannisyan H, Saus E, Ksiezopolska E, Hinks Roberts AJ, Louis EJ, Gabaldón T. 2020. Integrative omics analysis reveals a limited transcriptional shock after yeast interspecies hybridization. Front Genet 11:404. https://doi.org/10.3389/fgene.2020.00404.

58. Shi X, Ng DWK, Zhang C, Comai L, Ye W, Chen ZJ. 2012. cis- and transregulatory divergence between progenitor species determines geneexpression novelty in Arabidopsis allopolyploids. Nat Commun 3:950. https://doi.org/10.1038/ncomms1954.

59. Tirosh I, Reikhav S, Levy AA, Barkai N. 2009. A yeast hybrid provides insight into the evolution of gene expression regulation. Science 324:659-662. https://doi.org/10.1126/science.1169766.

60. de Jonge R, Bolton MD, Kombrink A, Van Den Berg GCM, Yadeta KA, Thomma BPHJ. 2013. Extensive chromosomal reshuffling drives evolution of virulence in an asexual pathogen. Genome Res 23:1271-1282. https://doi.org/10.1101/gr.152660.112.

61. Cook DE, Kramer HM, Torres DE, Seidl MF, Thomma BPHJ. 2020. A unique chromatin profile defines adaptive genomic regions in a fungal plant pathogen. Elife 9:e62208. https://doi.org/10.7554/eLife.62208.

62. Depotter JRL, Shi-Kunne $X$, Missonnier $H$, Liu T, Faino L, van den Berg GCM, Wood TA, Zhang B, Jacques A, Seidl MF, Thomma BPHJ. 2019. Dynamic virulence-related regions of the plant pathogenic fungus Verticillium dahliae display enhanced sequence conservation. Mol Ecol 28:3482-3495. https://doi.org/10.1111/mec.15168.

63. Klosterman SJ, Subbarao KV, Kang S, Veronese P, Gold SE, Thomma BPHJ, Chen Z, Henrissat B, Lee Y-H, Park J, Garcia-Pedrajas MD, Barbara DJ, Anchieta A, de Jonge R, Santhanam P, Maruthachalam K, Atallah Z, Amyotte SG, Paz Z, Inderbitzin P, Hayes RJ, Heiman DI, Young S, Zeng Q, Engels R, Galagan J, Cuomo CA, Dobinson KF, Ma L-J. 2011. Comparative genomics yields insights into niche adaptation of plant vascular wilt pathogens. PLoS Pathog 7:e1002137. https://doi.org/10.1371/journal .ppat.1002137.

64. de Jonge R, van Esse HP, Maruthachalam K, Bolton MD, Santhanam P, Saber MK, Zhang Z, Usami T, Lievens B, Subbarao KV, Thomma BPHJ. 2012. Tomato immune receptor Ve1 recognizes effector of multiple fungal pathogens uncovered by genome and RNA sequencing. Proc Natl Acad Sci U S A 109:5110-5115. https://doi.org/10.1073/pnas.1119623109. 
65. Kombrink A, Rovenich $H$, Shi-Kunne $X$, Rojas-Padilla $E$, van den Berg GCM, Domazakis E, de Jonge R, Valkenburg DJ, Sánchez-Vallet A, Seidl MF, Thomma BPHJ. 2017. Verticillium dahliae LysM effectors differentially contribute to virulence on plant hosts. Mol Plant Pathol 18:596-608. https://doi.org/10.1111/mpp.12520.

66. Lynch M, Conery JS. 2000. The evolutionary fate and consequences of duplicate genes. Science 290:1151-1155. https://doi.org/10.1126/science .290.5494.1151.

67. Sriswasdi S, Takashima M, Manabe R, Ohkuma M, Sugita T, Iwasaki W. 2016. Global deceleration of gene evolution following recent genome hybridizations in fungi. Genome Res 26:1081-1090. https://doi.org/10 $.1101 / \mathrm{gr} .205948 .116$.

68. Schranz ME, Mohammadin S, Edger PP. 2012. Ancient whole genome duplications, novelty and diversification: the WGD radiation lag-time model. Curr Opin Plant Biol 15:147-153. https://doi.org/10.1016/j.pbi .2012.03.011.

69. Maere S, De Bodt S, Raes J, Casneuf T, Van Montagu M, Kuiper M, Van de Peer Y. 2005. Modeling gene and genome duplications in eukaryotes. Proc Natl Acad Sci U S A 102:5454-5459. https://doi.org/10.1073/pnas .0501102102 .

70. Smukowski Heil CS, DeSevo CG, Pai DA, Tucker CM, Hoang ML, Dunham MJ. 2017. Loss of heterozygosity drives adaptation in hybrid yeast. Mol Biol Evol 34:1596-1612. https://doi.org/10.1093/molbev/msx098.

71. Hou J, Friedrich A, De Montigny J, Schacherer J. 2014. Chromosomal rearrangements as a major mechanism in the onset of reproductive isolation in Saccharomyces cerevisiae. Curr Biol 24:1153-1159. https://doi .org/10.1016/j.cub.2014.03.063.

72. Koren S, Walenz BP, Berlin K, Miller JR, Bergman NH, Phillippy AM. 2017. Canu: scalable and accurate long-read assembly via adaptive k-mer weighting and repeat separation. Genome Res 27:722-736. https://doi .org/10.1101/gr.215087.116.

73. Firtina C, Kim JS, Alser M, Cali DS, Cicek AE, Alkan C, Mutlu O. 2020. Apollo: a sequencing-technology-independent, scalable, and accurate assembly polishing algorithm. Bioinformatics 36:3669-3679. https://doi .org/10.1093/bioinformatics/btaa179.

74. Li H. 2013. Aligning sequence reads, clone sequences and assembly contigs with BWA-MEM. arXiv https://arxiv.org/abs/1303.3997.

75. Durand NC, Shamim MS, Machol I, Rao SSP, Huntley MH, Lander ES, Aiden EL. 2016. Juicer provides a one-click system for analyzing loop-resolution Hi-C experiments. Cell Syst 3:95-98. https://doi.org/10.1016/j .cels.2016.07.002.

76. Dudchenko O, Batra SS, Omer AD, Nyquist SK, Hoeger M, Durand NC, Shamim MS, Machol I, Lander ES, Aiden AP, Aiden EL. 2017. De novo assembly of the Aedes aegypti genome using $\mathrm{Hi}-\mathrm{C}$ yields chromosome-length scaffolds. Science 356:92-95. https://doi.org/10.1126/science.aal3327.

77. Dudchenko O, Shamim M, Batra S, Durand N, Musial N, Mostofa R, Pham M, Glenn St Hilaire B, Yao W, Stamenova E, Hoeger M, Nyquist S, Korchina V, Pletch K, Flanagan J, Tomaszewicz A, McAloose D, Pérez Estrada C, Novak B, Omer A, Aiden E. 2018. The Juicebox Assembly Tools module facilitates de novo assembly of mammalian genomes with chromosome-length scaffolds for under $\$ 1000$. bioRxiv https://doi.org/10.1101/254797.

78. Li H, Handsaker B, Wysoker A, Fennell T, Ruan J, Homer N, Marth G, Abecasis G, Durbin R, 1000 Genome Project Data Processing Subgroup. 2009. The Sequence Alignment/Map format and SAMtools. Bioinformatics 25:2078-2079. https://doi.org/10.1093/bioinformatics/btp352.

79. Li H. 2018. Minimap2: pairwise alignment for nucleotide sequences. Bioinformatics 34:3094-3100. https://doi.org/10.1093/bioinformatics/bty191.

80. Kim D, Pertea G, Trapnell C, Pimentel H, Kelley R, Salzberg SL. 2013. TopHat2: accurate alignment of transcriptomes in the presence of insertions, deletions and gene fusions. Genome Biol 14:R36. https://doi.org/ 10.1186/gb-2013-14-4-r36.

81. Juan J, Armenteros A, Tsirigos KD, Sønderby CK, Petersen TN, Winther O, Brunak S, Von Heijne G, Nielsen H. 2019. SignalP 5.0 improves signal peptide predictions using deep neural networks. Nat Biotechnol 37:420-423. https://doi.org/10.1038/s41587-019-0036-z.

82. Jones $P$, Binns D, Chang HY, Fraser M, Li W, McAnulla C, McWilliam H, Maslen J, Mitchell A, Nuka G, Pesseat S, Quinn AF, Sangrador-Vegas A, Scheremetjew M, Yong SY, Lopez R, Hunter S. 2014. InterProScan 5: genome-scale protein function classification. Bioinformatics 30:1236-1240. https://doi.org/10.1093/bioinformatics/btu031.
83. Huerta-Cepas J, Forslund K, Coelho LP, Szklarczyk D, Jensen LJ, Von Mering C, Bork P. 2017. Fast genome-wide functional annotation through orthology assignment by eggNOG-mapper. Mol Biol Evol 34:2115-2122. https:// doi.org/10.1093/molbev/msx148.

84. Huerta-Cepas J, Szklarczyk D, Heller D, Hernández-Plaza A, Forslund SK, Cook H, Mende DR, Letunic I, Rattei T, Jensen LJ, Von Mering C, Bork P. 2019. EggNOG 5.0: a hierarchical, functionally and phylogenetically annotated orthology resource based on 5090 organisms and 2502 viruses. Nucleic Acids Res 47:D309-D314. https://doi.org/10.1093/nar/ gky1085.

85. Zhang $H$, Yohe $T$, Huang L, Entwistle $S$, Wu P, Yang Z, Busk PK, Xu Y, Yin Y. 2018. DbCAN2: a meta server for automated carbohydrate-active enzyme annotation. Nucleic Acids Res 46:W95-W101. https://doi.org/10 1093/nar/gky418.

86. Yin Y, Mao X, Yang J, Chen X, Mao F, Xu Y. 2012. DbCAN: a Web resource for automated carbohydrate-active enzyme annotation. Nucleic Acids Res 40:W445-W451. https://doi.org/10.1093/nar/gks479.

87. Kurtz S, Phillippy A, Delcher AL, Smoot M, Shumway M, Antonescu C, Salzberg SL. 2004. Versatile and open software for comparing large genomes. Genome Biol 5:R12. https://doi.org/10.1186/gb-2004-5-2-r12.

88. Rice P, Longden I, Bleasby A. 2000. EMBOSS: the European molecular biology open software suite. Trends Genet 16:276-277. https://doi.org/ 10.1016/s0168-9525(00)02024-2.

89. Gu Z, Gu L, Eils R, Schlesner M, Brors B. 2014. Circlize implements and enhances circular visualization in R. Bioinformatics 30:2811-2812. https://doi.org/10.1093/bioinformatics/btu393.

90. Seppey M, Manni M, Zdobnov EM. 2019. BUSCO: assessing genome assembly and annotation completeness. Methods Mol Biol 1962:227-245. https://doi.org/10.1007/978-1-4939-9173-0_14.

91. Bao W, Kojima KK, Kohany O. 2015. Repbase Update, a database of repetitive elements in eukaryotic genomes. Mob DNA 6:11. https://doi.org/10 .1186/s13100-015-0041-9.

92. Katoh K, Standley DM. 2013. MAFFT multiple sequence alignment software version 7: improvements in performance and usability. Mol Biol Evol 30:772-780. https://doi.org/10.1093/molbev/mst010.

93. Stamatakis A. 2014. RAxML version 8: a tool for phylogenetic analysis and post-analysis of large phylogenies. Bioinformatics 30:1312-1313. https://doi.org/10.1093/bioinformatics/btu033.

94. Miele V, Penel S, Duret L. 2011. Ultra-fast sequence clustering from similarity networks with SiLiX. BMC Bioinformatics 12:116. https://doi.org/10 .1186/1471-2105-12-116.

95. Pignatelli M, Serras F, Moya A, Guigó R, Corominas M. 2009. CROC: finding chromosomal clusters in eukaryotic genomes. Bioinformatics 25:1552-1553. https://doi.org/10.1093/bioinformatics/btp248.

96. Webb AE, Walsh TA, O'Connell MJ. 2017. VESPA: very large-scale evolutionary and selective pressure analyses. PeerJ Comput Sci 3:e118. https://doi.org/10.7717/peerj-cs.118.

97. Edgar RC. 2004. MUSCLE: multiple sequence alignment with high accuracy and high throughput. Nucleic Acids Res 32:1792-1797. https://doi .org/10.1093/nar/gkh340.

98. Yang Z. 2007. PAML 4: phylogenetic analysis by maximum likelihood. Mol Biol Evol 24:1586-1591. https://doi.org/10.1093/molbev/msm088.

99. Grabherr MG, Haas BJ, Yassour M, Levin JZ, Thompson DA, Amit I, Adiconis X, Fan L, Raychowdhury R, Zeng Q, Chen Z, Mauceli E, Hacohen N, Gnirke A, Rhind N, di Palma F, Birren BW, Nusbaum C, Lindblad-Toh K, Friedman N, Regev A. 2011. Trinity: reconstructing a full-length transcriptome without a genome from RNA-Seq data. Nat Biotechnol 29:644-652. https://doi.org/10.1038/nbt.1883.

100. Langmead B, Salzberg SL. 2012. Fast gapped-read alignment with Bowtie 2. Nat Methods 9:357-359. https://doi.org/10.1038/nmeth.1923.

101. Robinson MD, McCarthy DJ, Smyth GK. 2010. edgeR: a Bioconductor package for differential expression analysis of digital gene expression data. Bioinformatics 26:139-140. https://doi.org/10.1093/bioinformatics/btp616.

102. Quinlan AR, Hall IM. 2010. BEDTools: a flexible suite of utilities for comparing genomic features. Bioinformatics 26:841-842.

103. Hunt M, De Silva N, Otto TD, Parkhill J, Keane JA, Harris SR. 2015. Circlator: automated circularization of genome assemblies using long sequencing reads. Genome Biol 16:294.

104. Simms D, Cizdziel P, Chomczynski P. 1993. TRIzol: a new reagent for optimal single-step isolation of RNA. Focus 15:99-102. 